\title{
Computational Methods for Stability and Control (COMSAC): The Time Has Come
}

\author{
Robert M. Hall $^{*}$ and Robert T. Biedron ${ }^{\dagger}$ \\ NASA Langley Research Center, Hampton, Virginia 23681 \\ Douglas N. Ball ${ }^{\star}$ and David R. Bogue ${ }^{\S}$ \\ Boeing Commercial Aircraft, Seattle, Washington 98055 \\ James Chung" and Bradford E. Green ${ }^{\text {ॠ }}$ \\ Naval Air Systems Command, Patuxent River, Maryland 20670 \\ Matthew J. Grismer ${ }^{* *}$ and Gregory P. Brooks ${ }^{\dagger \dagger}$ \\ Air Force Research Laboratory, Dayton, Ohio 45433 \\ Joseph R. Chambers ${ }^{*}$ \\ Chambers Consulting, Yorktown, Virginia 23692
}

\begin{abstract}
Powerful computational fluid dynamics (CFD) tools have emerged that appear to offer significant benefits as an adjunct to the experimental methods used by the stability and control community to predict aerodynamic parameters. The decreasing costs for and increasing availability of computing hours are making these applications increasingly viable as time goes on and the cost of computing continues to drop. This paper summarizes the efforts of four organizations to utilize high-end computational fluid dynamics (CFD) tools to address the challenges of the stability and control arena. General motivation and the backdrop for these efforts will be summarized as well as examples of current applications.
\end{abstract}

\section{Nomenclature}

$C_{D} \quad=$ drag coefficient

$C_{L} \quad=$ lift coefficient

$C_{L_{\alpha}} \quad=$ derivative of lift coefficient with $\alpha$

$C_{l} \quad=$ rolling moment coefficient

$C_{m} \quad=$ pitching moment coefficient

$C_{n} \quad=$ yawing moment coefficient

$C_{n \beta} \quad=$ directional stability

$C_{p} \quad=$ pressure coefficient

$C_{Y} \quad=$ side force coefficient

$c \quad=$ mean aerodynamic chord, $f t$.

$k=$ nondimensional frequency, $\omega c / 2 U_{\infty}$

\footnotetext{
* Senior Researcher, Research and Technology Directorate, MS 499, Associate Fellow AIAA.

$\dagger$ Senior Researcher, Research and Technology Directorate, MS 128, Member AIAA.

$\$$ Chief Engineer, Enabling Technology and Research, Member AIAA.

$\S$ S\&C Focal for Enabling Technology CFD, Enabling Technology and Research.

`Aerospace Engineer, Advanced Aerodynamics Br, Bldg 2187, Unit 5, Suite 1320-B, 48110 Shaw Rd, Senior Member AIAA

${ }^{* *}$ Senior Research Aerospace Engineer, AFRL/VAAC, Senior Member AIAA.

${ }^{\dagger}$ Research Aerospace Engineer, AFRL/VAAC, Member AIAA.

\#Consultant, 205 Old Dominion Rd.
} 


\begin{tabular}{|c|c|}
\hline$M_{\infty}$ & $=$ free-stream Mach number \\
\hline$R e_{c}$ & $=$ Reynolds number based on mean aerodynamic chord \\
\hline$U_{\infty}$ & $=$ free-stream velocity, ft/sec \\
\hline$\alpha$ & $=$ angle of attack, deg \\
\hline$\beta$ & $=$ angle of sideslip, deg \\
\hline$\delta_{s}$ & $=$ horizontal tail deflection, deg \\
\hline$\omega$ & $=$ dimensional frequency, $1 / \mathrm{sec}$ \\
\hline
\end{tabular}

\section{Introduction}

$\mathbf{R}$ egardless of expectations for range, cruise Mach number, or payload, if an airplane does not have acceptable - stability and control (S\&C) attributes, it will not go into production. Although the state of the art in prediction of aerodynamic S\&C parameters has vastly improved and the great majority of S\&C deficiencies identified and corrected during developmental wind tunnel testing, unsatisfactory S\&C characteristics are occasionally not identified before going to flight test. When these shortcomings do surface during flight test, however, they have often resulted in costly and inefficient problem resolution efforts with varying degrees of success. Many of the problems have been directly related to extremely complex aerodynamic phenomena and the difficulty in predicting and resolving such issues. The critical challenge for aerodynamicists is to reliably predict, and if necessary improve, all critical S\&C characteristics before getting to the flight test stage of development.

The wind tunnel has traditionally served as the primary tool to provide aerodynamic inputs for simulation data bases and to predict S\&C characteristics. Unfortunately, shortcomings exist in wind tunnel testing methods that can result in serious errors in the predicted S\&C characteristics. For example, most wind tunnel testing for $\mathrm{S} \& \mathrm{C}$ is conducted at subscale values of Reynolds number. Consequently, large effects of Reynolds number can occur with certain configurations because of the dependence of boundary-layer separation on Reynolds number. In the case of dynamic derivative testing, both low Reynolds numbers and low Mach numbers are typical due to facility constraints. As summarized by Wolowicz, Bowman, and Gilbert ${ }^{1}$, testing at reduced values of Reynolds and Mach numbers poses significant challenges. Model fidelity and model support interference can also be significant issues for wind tunnel testing.

It is the belief of the authors that the next significant improvement in the state of the art for predicting the S\&C characteristics of a new vehicle is underway at this time with the application of powerful computational fluid dynamics (CFD) tools. While the current time-accurate, Navier-Stokes solutions to typical S\&C problems are still too resource intensive for widespread use, these costs are expected to continue to fall, at least for the next decade, according to Moore's Law. ${ }^{2}$ Assessment and validation efforts are now timely so that validated tools will be available when computational costs have dropped to the point where such solutions would be reasonably affordable in a typical S\&C setting.

Applying high-end CFD codes with technology greater or equal to Reynolds-averaged Navier Stokes (RANS) to specific areas of S\&C interest before first flight can help focus the wind tunnel program and provide improved understanding of the flow physics encountered. Developing, assessing, and maturing appropriate CFD codes for use in S\&C analyses is the goal of the Computational Methods for Stability and Control (COMSAC) project at NASA Langley and a goal of the various organizations that coauthor this paper. The purpose of the current report is to present the reasons why this application of high-end computational tools is timely, to highlight the efforts that are underway in each organization, and to show how these efforts can synergistically accelerate the progress of validating the high-end codes for both simple and complex stability and control challenges.

\section{Historical Examples of Shortcomings in Predictions for Stability and Control}

Historically, many civil and military aircraft development programs have encountered critical S\&C deficiencies during early stages of flight test. Despite thousands of hours of wind tunnel testing, these surprises have occurred across the speed range from takeoff/landing to cruise, with especially challenging encounters at the fringes of the flight envelope, where separated flows dominate.

Examples of a number of high performance military aircraft that encountered transonic, uncommanded lateral motions during flight test are summarized in figure $1 .{ }^{3}$ The common experience of the aircraft in figure 1 is that 
critical S\&C deficiencies were uncovered in flight test. Consequently, fixes had to be quickly found under less than ideal conditions in a "cut and try" mode without the general benefit of flow diagnostics. In many cases, adding vortex generators or fences rectified the problem while, in other cases, performance limitations were simply accepted. Reliably predicting such unacceptable S\&C characteristics before building flight hardware could result in more efficient configuration optimization and could avoid costly program delays.

In addition to the foregoing high-performance military aircraft, a broader spectrum of aircraft that encountered S\&C surprises in flight test is shown in figure 2. The examples shown include the Boeing 767-400, where the addition of raked wing tips altered the stall break and necessitated the development of a new vortilon pattern. ${ }^{4}$ The Lockheed Martin C-130J experienced a wing drop, or roll-off, due to propeller-induced effects, which impacted product development of that aircraft. The experience with the Boeing 777 involved missed predictions concerning aft loading on the main wing and effectiveness of the horizontal tail in flight. ${ }^{4}$ The Lear 23 development program had the challenge of fixing an abrupt wing stall associated with leading-edge separation on approach. The NASA experience with the loss of the first X-43A stack configuration appeared to be the result of a cumulative sum of uncertainties in many of the predicted stability and control characteristics. Finally, the Boeing 737 Next Generation aircraft demonstrated a new, and unexpected, sensitivity of rigging of the inboard trailing-edge flaps that was not apparent in the older models. ${ }^{4}$ These revelations from flight test manifested themselves as increased time and cost of development testing, acceptance of undesirable performance limitations, or limitations in future growth potential.

On the basis of this past experience, it is clear that vehicles across the spectrum from high-performance military to commercial transports to business jets have all suffered from S\&C deficiencies first realized in flight test. Even more sobering is the fact that nearly all of the vehicles in figure 2 could be considered derivatives of very similar earlier versions of the configuration. As the industry moves away from derivative aircraft to more revolutionary vehicles, whether blended wing bodies, uninhabited vehicles, or vehicles with active control effectors, either more accurate predictive tools must be developed or even more "surprises" will undoubtedly be encountered.

\section{Bridging the Cultural Divide}

It is now hoped that CFD tools can be brought to bear on S\&C issues and complement the traditional wind tunnel data sources. However, for this next step to become a practical reality, improved communications between the $\mathrm{S} \& \mathrm{C}$ and computational communities must occur, even though they have very different backgrounds. Some of the cultural differences between these two communities are summarized in a tongue-in-cheek comparison in figure 3. During the past 10 to 15 years, the CFD community has generally been focused on performance predictions associated with attached flows. It has been able to focus on flow physics, which is a beneficial byproduct of the computations. It's primary concern about flow separation has involved predicting the value of $\alpha$ at which it might begin. The focus of the performance computations was typically forces such as lift and drag for one or two design conditions involving symmetric and steady flight with attached flow. Accuracies were expected to be good, and analysts sought to optimize the configuration for performance. Knowledge of S\&C was not required.

In contrast, the $\mathrm{S} \& \mathrm{C}$ community has had to worry about integrated aerodynamic forces and moments in all six degrees of freedom including the effects of aircraft motion. Since the S\&C community is responsible for the vehicle over its entire operating envelope, it has had to be concerned about both attached flow conditions as well as massively separated flow conditions at values of $\alpha$ up to and beyond stall and for large values of $\beta$. Symmetric flight is important but is only a portion of the flight envelope. Whereas high accuracy is appropriate and needed for performance predictions, the $\mathrm{S} \& \mathrm{C}$ community would sometimes be reassured just to know the algebraic sign of a parameter--for example, to know if the configuration is dynamically damped or propelling in pitch, roll, and yaw. To improve a configuration or to resolve unacceptable deficiencies, there have been very few guidelines and only minimal flow diagnostics available. Consequently, cut and try has been the typical approach, which depended heavily on the practitioner's experience base with similar configurations. Little or no knowledge of CFD has been required for the majority of these engineers.

If CFD tools are to be applied to S\&C problems, both the S\&C and the CFD communities will have to actively be involved. It will be critical that the $\mathrm{S} \& \mathrm{C}$ community define levels of accuracy needed for both static 
and dynamic derivatives for a given vehicle. If accuracies can be relaxed relative to performance computations, then computational resources and, therefore, cost can be minimized assuming that the CFD community can quantify its uncertainty levels. Also, the S\&C community must help focus the application of the computational efforts. While wind-tunnel testing is designed to provide large data bases that can cover most of the flight envelope, high-end computations are resource intensive and can currently only be strategically applied. Finally, technical trust must be developed between the two communities. The CFD community will have to demonstrate that it can predict results in a timely way and without prior knowledge of the answer if its results and tools are to be accepted by the S\&C community for any role beyond providing flow diagnostics. Also, the S\&C community will need to become educated and conversant with CFD tools designed to be user friendly.

\section{IV.Recognizing the Need for CFD Tools}

During November of 2002, a NASA/DoD/Industry Flight Prediction Workshop sponsored by NASA and DoD was held in Williamsburg, Virginia. The invitation-only meeting was attended by 85 experts who shared critical experiences and involving the state-of-the-art in aerodynamic flight prediction. During break-out sessions, stability and control deficiencies were highlighted as high priority and the lack of robust, accurate computational tools as an adjunct to wind tunnel testing were also cited. These conclusions were used to help launch the current COMSAC effort.

In September of 2003, a follow-up Computational Methods for Stability and Control (COMSAC) Symposium sponsored by NASA was held in Hampton, Virginia with approximately 100 attendees. Thirty-four presentations were given including keynote and overview presentations from government and industry representatives as well as technical papers outlining the state of the art from government, industry, and academia. ${ }^{5,6}$ Both computational and $\mathrm{S} \& \mathrm{C}$ perspectives were presented. The challenge of working cooperatively while protecting proprietary concerns was discussed.

During the course of the COMSAC Symposium, it became clear that a high level of interest exists in pursuing the application of high-end codes to S\&C challenges. Coupled with the already existing shortfalls in wind tunnel capabilities is the growing realization that wind tunnel availability may be more limited in the future as a number of existing facilities are being considered for closure. All these factors were stimulating interest in accelerating the application of CFD tools to S\&C problems. It was also clear that many organizations are already actively pursuing interactions between these two disciplines.

However, several concerns were also expressed by attendees and speakers at the COMSAC Symposium. The first concern was that CFD had been "over marketed" on many occasions in the past and that, understandably, the S\&C community was skeptical of current capabilities in view of the extremely challenging separated flows encountered in its efforts. Also, the $\mathrm{S} \& \mathrm{C}$ community is aware that many current diagnostic computations are made with the code practitioner knowing in advance the answer and, therefore, able to optimize the code results through grid modifications or turbulence model selection. They wanted to see more "blind" predictions. The second major concern was that the high-end codes using RANS appear to produce incorrect answers for a large fraction of the $\mathrm{S} \& \mathrm{C}$ cases undertaken.

In summary, the symposium brought together two different engineering cultures for in-depth discussion of opportunities, challenges, and experiences with CFD methods for S\&C. Agreement existed in both cultures that applications of high-end computational tools to current $\mathrm{S} \& \mathrm{C}$ challenges for calibration and validation would be a milestone goal. Currently, it is obvious that this validation is very much in its infancy and that a considerable amount of work comparing computational predictions for many different configurations to actual flight, or wind tunnel, data will be required before these tools will be fully accepted by the S\&C community.

\section{COMSAC Project Objective}

Based on the COMSAC Symposium and other NASA/industry/government interactions, the objective for the COMSAC effort might best be stated as "accelerating the application, validation, and focused development of CFD methodology to S\&C aerodynamic predictions and analyses by coordinating industry/government efforts." The benefits of this effort are envisioned to include (1) better understanding and control of critical flow physics,

4

American Institute of Aeronautics and Astronautics 
(2) reduced and focused wind-tunnel and flight tests, and (3) risk reduction for future programs because of fewer surprises in flight test and certification. An informal COMSAC community was formed by NASA Langley, Naval Air Systems Command (NAVAIR), Boeing Commercial Airplanes, and the Air Force Research Laboratory (AFRL). While it is fully understood that many other industry and other government entities are involved in the pursuit of applying high-end computational codes to S\&C problems, the above listed organizations are attempting, through informal information sharing, to accelerate the process.

\section{A. NASA Langley}

\section{Status of Efforts}

The Langley approach has been to apply several mature, state-of-the-art computational codes to the respective problems of interest to explore differences resulting from the respective implementations of algorithm, turbulence modeling, and other factors. The unconventional Blended Wing Body (BWB) configuration has been the focus of the effort because of previous NASA/Boeing research and current interest in this advanced configuration. While the BWB has undergone several configuration evolutions over the past decade, the configuration being analyzed for the present work is the 450-1L configuration. ${ }^{7,8}$ As seen in figure 4, this flying-wing type configuration has a relatively thick center section with conventional outer wing panels. The three-engine installation incorporates pylon-mounted nacelles although research has also been accomplished in the past for boundary-layer ingestion inlets as well'.

The COMSAC BWB computational efforts have been underway since February of 2004 and are compared to Langley wind tunnel data which were intended to populate a simulation data base. Unfortunately, the existing experimental data sets, (particularly for the pitching moment coefficient) are all influenced in varying degrees by the presence of the model-mounting system. The photographs shown in figure 4 illustrate a .03 -scale model of the BWB mounted on two examples of the post supports used during the Langley 14- x 22-Foot Subsonic Tunnel (ST) and a 0.02-scale model of the BWB on a blade support in the Langley National Transonic Facility (NTF). The post mounts in the ST varied from bottom posts entering the model vertically through its belly region with different post diameters to a post entering the top, back of the model. All of the supports generated aerodynamic interference increments in the experimental data to some degree, as may be seen in Figure 5, which shows the variation in pitching moment measured in the NTF and on large and small posts in the 14- $\mathrm{x} 22-\mathrm{ft}$ ST. Although the NTF data and the large-post 14- x 22-ft ST data are for the same nominal Reynolds number (3.7 million), the small-post 14- x 22-ft ST data are at a slightly lower Reynolds number (2.5 million). To help gauge the relative effects of Reynolds number vs. post-size, Figure 5 also shows the large-post 14- x 22-ft ST at a Reynolds number of 2.7 million. While there is a noticeable effect due to Reynolds number differences, which were created by changing tunnel velocity, the post size has a far greater effect at virtually all values of $\alpha$.

The knowledge that mounting support interference was an issue was identified during the early $14-\mathrm{x} 22$-Foot ST testing program and led to computational corrections for the large post experimental data by Boeing Huntington Beach. Huntington Beach also designed the blade support for the NTF test to minimize interference effects. The COMSAC effort at Langley is continuing the effort of Huntington Beach to quantify the impact of the various support systems used so that the wind tunnel data can more easily be used to validate the CFD codes.

\section{Static computations}

Before discussing the correlation of CFD and experimental results for the BWB configuration, a brief discussion of pertinent $\mathrm{S} \& \mathrm{C}$ interests might serve to provide the reader with important background. Shown in Figure 6 is one of the experimental pitching moment curves from figure 5 and can be used to illustrate three key regions for $\mathrm{S} \& \mathrm{C}$. For the lower angles of attack near $0^{\circ}$, the pitching moments are generally linear with increasing AOA and the slope is negative, indicative of satisfactory longitudinal stability; such trends are usually exhibited for attached-flow conditions. Within the second AOA regime wing stall has resulted in shifting of the aerodynamic center of the configuration. This region is initiated by an abrupt longitudinal instability (pitchup) indicated by a reversal of slope to a positive trend and extends over the region of complex vortical and other separated flows. Finally, the third AOA regime occurs when the complete configuration stalls and the pitching moments return to a stable slope. The $\mathrm{S} \& \mathrm{C}$ analyst is concerned with the stability trends shown in each of the regimes - especially to the abruptness of instabilities and the proximity to the intended flight envelope as well as recovery from post-stall conditions. 
For all of the Langley computations to be presented, no attempt to model the tunnel walls has been made and the results are considered to be appropriate for "free air." Furthermore, initial computations did not account for the presence of the mounting supports while later computations, as will be shown, did. Most attention has been focused on capturing the longitudinal pitching moment coefficient, as this is critical to characterize longitudinal stability and has historically been difficult to accurately predict with CFD. In addition to comparison with experimental data, most cases have been run with at least two CFD codes for code-to-code comparisons. While by no means a true validation process, having two different codes giving nominally the same result leads to additional confidence in the computations that might otherwise be absent; on the other hand, large differences in computed results would suggest the need for further investigation.

With the understanding that the experimental data contain support interference while the initial CFD computations do not, the computational pitching moment results from the unstructured-grid solvers FUN3D ${ }^{10}$ and $\mathrm{COBALT}^{11}$ as well as the structured-grid solver PAB3D ${ }^{12}$ are compared to experimental data in Figure 7 . This figure shows a comparison of experimental data for $\mathrm{Re}_{c}$, Reynolds number based on mean aerodynamic chord, equal to 3.7 million, and for $\mathrm{M}_{\infty}=0.2$. The FUN3D and COBALT results were generated using the SpalartAllmaras ${ }^{13}$ (SA) turbulence model, while the PAB3D results were generated using the Girimaji ${ }^{14}$ explicit algebraic stress model. Additional static computations obtained with PAB3D and FUN3D, with comparison to experiment, can be found in reference 15 .

The results from COBALT tend to agree closer with the NTF data in terms of stability levels at lower values of $\alpha$ than the FUN3D predictions, but both capture the overall trends very well to the higher values of $\alpha$. The results shown correspond to the finest meshes used in the two solvers; COBALT is cell-based, so the number of cells is the proper measure of the grid size, while FUN3D is node based, thus the number of nodes indicates the problem size. More variation is seen between the two unstructured solvers and the structured solver, which shows a much lower level of stability at lower values of $\alpha$. It should be noted that the PAB3D results were for a coarser grid (700,000 cells) and with a different turbulence model. The data set from the 14- x 22-ft ST was taken with a large post support mounted through the belly of the model, and although displaced with a positive increment of moment, the trends (stability level at lower values of $\alpha$ and break points) agree with the NTF data. Since the NTF data utilized a lower surface blade support, which was designed to minimize support interference, it is encouraging that the CFD results fall closer to the NTF data than they do to the 14- $\mathrm{x} 22$-ft ST. However, the large differences in the experimental values, due to different levels of support interference, preclude any real validation of the computational data without modeling the respective support systems.

Because the experimentally measured dynamic data discussed in the next section were of necessity taken at very low dynamic pressures, a similar comparison of CFD and experimental static moment coefficients was repeated for $\mathrm{Re}_{\mathrm{c}}=700,000$, corresponding to the flow conditions for the dynamic tests. The grids used were the same as that for the higher Reynolds number, with the exception that the COBALT results shown were using a coarser mesh that was subsequently used for the majority of the dynamic pitch computations run with COBALT. This grid was chosen based on running three different grid resolutions at the higher Reynolds number (static) and by running two dynamic cases with the three grids. The experimental measurements were taken using a mounting system that allowed the models to be mounted either from the top or the bottom; both experimental data sets are shown in Figure 8. The differences between the experimental data sets at lower values of $\alpha$ for the two post mounts are unsettling in that the top mount shows pitch instability near an angle of attack of $0 .^{\circ}$ The bottom mount experimental data and the computations agree better.

More recently, CFD results have been obtained with the inclusion of the large-post mounting system of the 14- $\mathrm{x}$ 22-ft ST and with the blade-mounted system of the NTF. Starting with the mounting post effects in the 14- $\mathrm{x}$ 22-ft ST, figure 9 shows a plot of $\mathrm{C}_{\mathrm{m}}$ vs. $\alpha$ computed using FUN3D, albeit with a different, coarser grid than used in figure 7. Computed results are shown with and without the post, but in both cases the effect of tunnel walls are not included. Clearly the inclusion of the post has shifted the moment coefficient in the right direction, and by the right amount. The computed moment coefficient (both with and without post) shows a much broader plateau than the experiment around $\alpha=15^{\circ}$, or, for that matter, broader than the previous computation without post on the 4.9 million node grid; this is believed to be a consequence of the relatively coarse mesh. Simulations with a finer mesh are currently underway, as are computations including both the post and the tunnel walls. 
Similarly, interference effects were examined for the NTF mounting system. In this case, only the blade was modeled (the part of the support visible in Figure 4), not the large arc-sector located further downstream that supports the blade. Furthermore the tunnel walls were not modeled. Figure 10 shows the results computed using FUN3D with and without the blade, along with the NTF data. Clearly the inclusion of the blade moves the CFD results into better agreement with the data, though, the stability level prior to the lift break $\left(\alpha=8^{\circ}\right.$ or $\left.9^{\circ}\right)$ still appears to be over estimated.

All the results presented above were for low subsonic Mach numbers. Transonic flows have also been considered. Figure 11 shows comparison of CFD results, including the NTF mounting blade, computed using FUN3D and the unstructured solver USM3D ${ }^{16}$, at conditions corresponding to an NTF test at Mach 0.85 and a $\operatorname{Re}_{\mathrm{c}}$ $=10$ million. Results from both codes were generated using the same mesh; note however that since USM3D is a cell-based code, the effective resolution of the mesh is approximately six times greater for USM3D than for FUN3D. Both codes capture the stability level within the linear range of pitching moment very well, while FUN3D predicts an early (relative to experiment) pitch break near $\alpha \sim 5^{\circ}$, and USM3D predicts a late pitch break at $\alpha \sim 6^{\circ}$.

A final example of static computations are shown in figure 12, which is an example of computations for the BWB model tested in the 14- x 22-ft ST in sideslip. For this case, the mounting support was not included in the CFD computation. As seen in the results, the predicted computational slope of rolling moment coefficient versus sideslip is in good agreement with experiment for values of $\beta>5^{\circ}$. Additional analysis would be required to determine if sting effects have influenced the rolling moments for $\beta \sim 0^{\circ}$.

\section{Dynamic computations}

Pitch damping for the BWB was chosen as the highest priority dynamic derivative to be computed because of the criticality of longitudinal motions for flying-wing configurations. Unfortunately, the national capability to measure pitch damping in wind tunnels is very limited, and is usually conducted at low Reynolds and Mach numbers. Consequently, the team embarked on time-accurate computations simulating forced oscillations in the longitudinal plane, where the model is oscillated at a fixed sinusoidal frequency about a mean angle of attack. The resulting variation of pitching moment in this condition quantifies the aerodynamic pitch damping derivative, which is a combination of terms due to pitch rate and rate of change of angle of attack.

Load limitations on the wind-tunnel forced oscillation rig used to obtain the experimental data meant that the tests had to be conducted at a low dynamic pressure corresponding to $\mathrm{Re}_{\mathrm{c}}=700,000$. Data were taken in the $14-\mathrm{x}$ $22-\mathrm{ft}$ ST over a range of mean angles and for several values of pitch amplitude and reduced frequency. For the range of mean angles considered here, the corresponding experimental data were taken with the model mounted from the bottom. The majority of the computations were carried out for pitch amplitudes of plus or minus $5^{\circ}$, and for a reduced frequency, $\mathrm{k}$, of 0.07 . None of the force-oscillation computations attempted to date have included the post or tunnel walls. For these data curves, the S\&C analyst is interested in the overall character of the data trend. If the trend of pitching moment with $\alpha$ oscillations results in a counter-clockwise variation of pitching moment, the configuration is damped. If, however, the loop exhibits a clockwise trend, the configuration will be undamped (propelling) for that condition. The analyst is particularly interested in the magnitude of pitching moment at the time of positive and negative pitch motions at the mean value of $\alpha$ since this defines the magnitude of the pitch damping derivative for that particular value of $\alpha$. It should be noted that for all of the plots to be shown in this section the loops are counter-clockwise yielding pitch damping with the exception of one plot, which will be discussed further.

As these time-accurate computations were attempted, it became apparent that a real challenge was to determine the appropriate balance between grid resolution and temporal resolution for this class of problems. As the grid resolution becomes finer, the time step requirement for temporal convergence can become more demanding as well. Clearly, even with the increasing availability of computer resources, there is a prudent engineering limit to the amount of resources to devote. None of the results obtained to date are believed to be grid converged in a strict sense although they are believed adequate from an engineering sense. The time step 
refinement studies suggest that the results presented below are reasonably well converged temporally for the grids used. As for the static moment cases, several codes were employed: COBALT, FUN3D, and USM3D. Further details of the FUN3D computations may be found in reference 17, while reference 18 presents the COBALT computations in greater detail. In particular both references discuss mesh and time step studies that were used to determine practical mesh and time step ranges for subsequent computations with each code.

Figure 13 shows a comparison of the simulated forced-oscillation results computed with the three codes for a mean angle of $0^{\circ}$. USM3D and FUN3D used the Spalart-Allmaras (SA) model while COBALT utilized Menter's (SST) model. ${ }^{19}$ The shapes of all three computed curves are similar to the wind-tunnel data in some respects and different in other respects; it is difficult to judge which is "better" overall.

Both the COBALT and FUN3D codes have been used to obtain solutions for mean values of $\alpha$ of $0^{\circ}, 8^{\circ}, 16^{\circ}$, $24^{\circ}$, and $32^{\circ}$. Figure 14 shows a summary plot for FUN3D wherein the computed dynamic data for various mean angles are shown in different colors on top of the computed static pitching moment curve, with a similar treatment of the experimental data. For the pitch amplitude and reduced frequency considered, the general shape of the dynamic pitching moment curve is heavily influenced by the local shape of the static moment curve--which is, in turn, shaped by the flow separation states. Although not shown, the corresponding plot for the COBALT results would show a very similar trend.

Detailed results from both codes, for mean angles of $8^{\circ}$ and $24^{\circ}$ are shown in figures 15 and 16 respectively. Generally speaking, the shape of the computed dynamic pitching moment curves agree well with the data for a mean angle of $8^{\circ}$, but the agreement has degraded considerably by $16^{\circ}$, as seen in figure 14 for the loop centered at $\alpha=16^{\circ}$. Note that between $\alpha=14^{\circ}$ and $22^{\circ}$, the experimental static curve in figure 14 exhibits an essentially constant static pitching moment, while the computed static pitching moment (from both codes) has a slight negative slope. At a mean angle of $24^{\circ}$, any semblance of the computed data to the experimental data has vanished, as seen in figure 16. The experimental curve has collapsed near the mean angle, indicating a degradation of pitch damping at that value of $\alpha$. Both computed RANS results show wide loops without significant loss of pitch damping. Note also that the experimental static pitching moment coefficient in figure 14 over the range of $22^{\circ}$ to $28^{\circ}$ has a much steeper negative slope than the computed static curve. At $\alpha=32^{\circ}$, however, the decrease in pitch damping has disappeared from the experimental data and reasonable agreement between computation and experiment is once again observed. It is also observed that beyond $30^{\circ}$, the computed and experimental static $\mathrm{C}_{\mathrm{m}}$ curves have nearly the same negative slope.

To further investigate the poor comparison between experiment and data at $24^{\circ}$, COBALT was used to perform computations using DES. ${ }^{20}$ Extensive experience with the DES model in COBALT suggested that in many high-angle of attack situations, DES, which has inherently less dissipation in off-body separated regions than RANS, leads to better agreement with measured data. Since for DES (in the LES region) modeling errors decrease as the grid is refined, the original mesh was adapted (based on vorticity) to cluster points in the off-body separation region. The SA-based DES results on the adapted mesh are shown in figure 17, as are the RANS results on the original mesh (i.e. the same results shown in figure 16), and DES results on the original mesh. In the interest of clarity, phase average data of the DES computations are shown, rather than the complete instantaneous data set. It should be noted that the DES calculations, while on a per-time-step basis are no more expensive to compute than RANS on the same mesh, require perhaps an order of magnitude more time steps before meaningful phase-averaged data can be obtained. The fact that the phase-averaged DES data shown figure 17 still have some high frequency content indicate that more times steps are really needed before computing the phase averages; nonetheless, the trend that DES is giving results more representative of the experiment is unmistakable. Also in the interest of clarity, SST RANS results on the adapted mesh are not shown. Only the DES results on the adapted mesh show a decrease in pitch damping; the RANS computations on the same adapted mesh do not.

As an additional note to the discussion about the DES results given in figure 17, the experimental wind-tunnel data also have frequency content that has to be smoothed by averaging 40 or 50 cycles of data. Consequently, the high-frequency content of the DES solution may be physical and realistic. If the comparisons of figure 17 are representative, expending the resources to do the loop averaging for DES may be a requirement, even if it is an expensive requirement at this time. 


\section{Summary}

While the computational effort at Langley has been underway for a relatively short time, a good deal of progress has been made in advancing the understanding of the requirements for using CFD as a tool for S\&C. When the work began, the dramatic influence of the support interference on the close-coupled BWB configuration was not fully appreciated. However, the results obtained to date suggest that if the support is modeled by the computation, then the agreement of the computed static pitching moment coefficient with the experimental data can be improved considerably. Still to be investigated is whether or not modeling the support when performing dynamic simulations (considerably more difficult and expensive) will yield better agreement with the measured data.

Determining grid and time-step convergence for unsteady flows is extremely resource intensive. Relatively speaking, it is far easier to investigate time-step convergence since a halving of the time step simply doubles the compute time and (in the asymptotic region) leads to a quartering of the temporal error for the typical second order schemes used. In contrast, to quarter the error in the second-order spatial discretization used in most "production" CFD codes requires that the mesh size be halved in each direction, which leads, in the case of structured grids, to a factor of 8 increase in the computing time. Compounding the problem, finer meshes typically require smaller time steps for temporal convergence, so one is easily faced with a factor of 16 increase in resource requirements to reduce the error by a factor of four. In this sense, grid convergence is the tall pole in tent, and as mentioned, it is unlikely that the results for the BWB obtained to date are grid converged. Nevertheless, it is always important to address grid convergence and it will be discussed further in the status report from AFRL.

\section{Acknowledgements}

The Langley authors would like to thank the following colleagues for providing computational and experimental results: Jim Forsythe, Cobalt Solutions; Neal Frink, Ray Mineck, S. Paul Pao, Mike Fremaux, and Dan Vicroy, all NASA Langley Research Center. Thanks and appreciation are also due to our Boeing Huntington Beach colleagues, including Norm Princen, Dhar Patel and Doug Friedman.

\section{B. Naval Air Systems Command (NAVAIR)}

The nature of today's Defense systems acquisition process demands the procurement of increasingly complex systems in shorter time periods with less overall funding. In particular, the test and evaluation (T\&E) community is increasingly challenged to reduce overall test time yet not diminish their mission of comprehensive independent assessment of the warfighter's weaponry. This challenge becomes increasingly difficult without the ability to employ advanced technologies aimed at assisting the tester in performing their mission.

The most promising approach to support this acquisition challenge is virtual testing through the use of Modeling and Simulation (M\&S). The benefits of M\&S are not new, but model validation, computing access and speed, and efficient tester interface with multi-disciplinary $M \& S$ systems are enabling $M \& S$ to more effectively support testing programs.

Under the auspices of the DoD High Performance Computing (HPC) Modernization Office, Naval Air Systems Command (NAVAIR) is managing a tri-service software development program that is aimed at integrating multiple disciplines through the linking of various codes under a single framework called STEPNET (Simulation, Test and Evaluation Process NETwork). These codes and respective application models are then to be employed by the engineer to solve any one of a set of aeromechanical problems. The umbrella for the current work is the Collaborative Simulation and Testing (CST) Portfolio. CST is an integrated portfolio of high performance computing codes directly and specifically aimed at bridging the gap between high-end M\&S capabilities and test program needs. It is focused on "applied" high-fidelity M\&S that makes advancements in model validation, creates the ability for data to be quickly processed for "near-time" (as opposed to "real-time", or "near real-time") support of test programs, and enables effective communication interfaces between test engineers and the high-fidelity M\&S front-end. Funding for CST comes from the DoD HPC Modernization Program's Common High Performance Software Support Initiative (CHSSI) and is managed by Dr. Dave Findlay. 
Computational Fluid Dynamics (CFD), wind tunnel tests, flight dynamics analysis tools, and flight simulation programs have been used separately and independently of the T\&E process in the past. The goal of the sub-project "Integrated Simulation of Air Vehicle Stability and Control for Test and Evaluation" is to integrate CFD analysis with flight simulation to demonstrate an improved T\&E process for fixed-wing aircraft. CFD methodology, based on the NASA Langley-developed USM3D CFD code (part of an existing state-of-the-art computational system, known as TetrUSS ${ }^{21}$ ) in conjunction with NAVAIR-developed CASTLE flight simulation code, will be used for the flight test planning and data validation/evaluation process. This project is expected to provide a diagnostic tool for flight test decision makers and contribute to the reduction of risks, integration between modeling and simulation and testing, and closer coupling of different technical areas.

The joint NAVAIR/NASA commitment provides a substantial synergy for improving the capabilities of TetrUSS, to enhance its ability to solve important problems for stability and control. A primary thrust of this project is the efficient computation of dynamic stability derivatives across an aircraft flight envelope for input into flight simulation programs. One fundamental requirement is for TetrUSS to be a validated, useable, robust system for computing unsteady, often massively separated flows on complex military and civilian aircraft configurations in dynamic motion with a known range of uncertainty. Work has been performed to enhance, customize, and validate TetrUSS capabilities toward ready computation of dynamic derivatives across the flight envelope. The primary support includes the implementation and validation of unsteady Reynolds-Averaged Navier-Stokes (RANS) and Detached Eddy Simulation (DES), a forced oscillation and 6-DOF free-to-roll (FTR) analysis capability, low-Mach number preconditioning, utilization of advanced two-equation turbulence models, Chimera overset moving grids, and assessments of code scalability across multiple HPCMO computer platforms. Work is well underway for each of the above capabilities. ${ }^{22,23}$

The examples to be illustrated for this status report are from analyses of the S\&C characteristics of the F/A$18 \mathrm{E} / \mathrm{F}$. This aircraft, in its pre-production version, experienced abrupt wing stall which resulted in its susceptibility to asymmetric wing stall, or wing drop. ${ }^{24}$ Because of the abrupt wing stall of pre-production aircraft, this version makes a good test bed for code comparisons and will be used for the current investigation. The wing drop on the pre-production aircraft was eliminated by modifying the leading-edge flap schedule and adding a porous surface over the wing-fold fairing. The production F/A-18E/F, which is being delivered to the fleet today, does not have wing drop.

The goal of the present study was to assess the ability of CFD to predict the longitudinal and lateral/directional $\mathrm{S} \& \mathrm{C}$ characteristics of the pre-production F/A-18E at transonic Mach numbers without prior knowledge of existing wind tunnel or flight test data. Executing the computations in the "blind" would address one of the main concerns of the traditional S\&C community that the CFD engineer often knew the answer before doing the computation while simultaneously educating CFD users about the needs of the S\&C community. The study summarized in this paper is described fully in a reference by Green. ${ }^{25}$

The geometry evaluated during this computational study was that of the 0.08 -scale pre-production $\mathrm{F} / \mathrm{A}-18 \mathrm{E}$ wind tunnel model. A picture of the computational geometry is shown in figure 18. The wing was modeled with $6^{\circ} / 8^{\circ} / 4^{\circ}$ flaps, indicating that the leading-edge flap was deflected $6^{\circ}$, the trailing-edge flap was deflected $8^{\circ}$ and the aileron was deflected $4^{\circ}$ downward. The geometry was modeled with both horizontal and vertical tails present. Horizontal tail deflections, $\delta_{\mathrm{s}}$, of $-6^{\circ}, 0^{\circ}$ and $6^{\circ}$ were evaluated for the longitudinal study, while $\delta_{\mathrm{s}}=0^{\circ}$ was used for the lateral/directional study. A Sidewinder missile and launcher were modeled at the wing tip and the inlet was modeled as flow-thru. The aft-body distortion required for sting mounting of the 0.08 -scale wind tunnel model was included in the computational geometry as was the presence of the sting itself. The sting geometry was truncated at $50 \%$ fuselage length behind the nozzle.

\section{Grid Assessment}

Grids with four different fidelities were used during this study. Coarse, medium and fine grids, see figure 19, were used for the predictions of the longitudinal stability and control characteristics. In these three grids, only half of the aircraft was modeled and a symmetry-plane boundary condition was implemented. All of the grids generated during this study had viscous tetrahedral cells near the surface and inviscid tetrahedral cells away from the surface. The y-plus value of the first cell above the surface was approximately unity for each of the grids. The coarse grid used 6.5 million cells but had minimal resolution of the verticals tails and the horizontal tail. The 
medium grid with 11.5 million cells was constructed to bring the resolution of the verticals and horizontal up to the level of the wing used in the coarse grid. The fine grid, at 15.1 million cells, started with the medium grid and enriched the gridding over the wing. A fourth, full grid for lateral/directional calculations, not shown in figure 19, was originally intended to be twice that of the medium grids, or 23 million cells. This, however, turned out to be too resource intensive and a compromise between the coarse and medium grids was achieved which yielded a total of 16.2 million cells. The lateral/directional grid was generated with $\delta_{\mathrm{s}}=0^{\circ}$.

Since the CFD calculations were to be performed without any prior knowledge of existing wind tunnel or flight test data, a grid resolution study was performed to determine the size of the grid necessary to accurately calculate the forces and moments on the pre-production F/A-18E. Accurately predicting the longitudinal characteristics of the aircraft was the main objective of performing the grid refinement study. In this section, the results of the grid refinement study at $\mathrm{M}_{\infty}=0.8$ and 0.9 are presented in figure 20, where lift and pitching moment coefficients are presented as a function of angle of attack for the coarse, medium, and fine grids.

For the $\mathrm{M}_{\infty}=0.8$ results, the coarse grid results are very similar to those of the medium and fine grid results, although the pitching moment coefficient of the coarse grid near $\alpha=10^{\circ}$ is slightly more negative than that of the medium and fine grids. Since values of $\delta_{\mathrm{s}}=-6^{\circ}$ and $6^{\circ}$ were going to be evaluated, it was anticipated that this difference in pitching moment could become larger for the non-zero tail deflections. As a result, the medium grid was used for the calculations with $\delta_{\mathrm{s}}=-6^{\circ}$ and $6^{\circ}$ at $\mathrm{M}_{\infty}=0.8$. The sudden slope change in the lift and pitching moment curves in figure 20 at $\alpha=6^{\circ}$ are indicative of the abrupt stall phenomenon that the pre-production F/A$18 \mathrm{E}$ experienced.

For the $\mathrm{M}_{\infty}=0.9$ results, see figure 20 again, the lift and pitching moment coefficients for the coarse, medium and fine grids are very similar. However, the onset of stall for the fine grid is $1^{\circ}$ lower in $\alpha$ than that for the coarse and medium grids. As a result, there is a significant difference in the lift and pitching moment coefficients at $\alpha=7^{\circ}$ resulting in earlier instability (positive slope of pitching moment vs. $\alpha$ curve). Without prior knowledge of the wind tunnel data, it was assumed that the solutions generated with more points on the wing would be more accurate than those solutions with less resolution on the wing (even though wind tunnel data would later suggest otherwise). As a result, the fine grid was used to analyze the configurations with $\delta_{s}=-6^{\circ}$ and $6^{\circ}$ at $\mathrm{M}_{\infty}=0.9$. It is understood that even with the fine grid, the solutions cannot be considered grid resolved.

\section{Longitudinal Stability and Control}

The longitudinal stability and control predictions for $\mathrm{M}_{\infty}=0.8$ are compared to wind tunnel data from the NASA Langley Research Center in Hampton, VA, NASA Ames Research Center in Moffett Field, CA and CALSPAN in Buffalo, NY. (More details are again given in the paper by Green. ${ }^{25}$ ) In figure 21 both lift coefficient and pitching moment coefficients are summarized for the 3 horizontal tails settings. While all three experimental entries generated data for $\delta_{\mathrm{s}}=0,{ }^{\circ}$ only the Ames entry generated data for the non-zero deflection angles. Consequently, the experimental data for $\delta_{\mathrm{s}}=0^{\circ}$ gives insight into the differences between wind tunnel entries in different tunnels and are an indicator of the uncertainty in the wind tunnel data due facility and installation differences. The CFD results compare well with the wind tunnel data between $-2^{\circ}<\alpha<6^{\circ}$. However, the CFD appears to predict an earlier stall onset at $\alpha=7^{\circ}$ than the wind tunnel data indicates. Between $8^{\circ}<\alpha<12^{\circ}$, CFD predicts an approximately linear variation of lift whereas the wind tunnel indicates otherwise. The reasons for these discrepancies are not known, but may be related to the unsteady nature of the abrupt wing phenomena. Importantly from an S\&C perspective, the trends and increments for the horizontal tail deflections are well represented in the computations.

The corresponding pitching moment coefficients are also presented in figure 21 and tell a similar story. First, stall seems to be reflected at a lower value of $\alpha$ than in the wind tunnel. However, increments and general shapes of the curves for the different horizontal tail deflections are well represented. Green ${ }^{25}$ does note that the code predicts less stable longitudinal stability characteristics between $-2^{\circ}<\alpha<6^{\circ}$. 


\section{Lateral/Directional Stability Predictions}

The lateral/directional predictions were performed with the appropriate full-span grid of 16.2 million cells. As mentioned already, this full-span grid is more coarse than the medium grid used for the longitudinal predictions. In figures 22 to 24 , the rolling moment, yawing moment and side force coefficients for the CFD calculations and the wind tunnel data are plotted as a function of $\beta$ for $\mathrm{M}_{\infty}=0.9$ for values of $\alpha=5^{\circ}, 7^{\circ}$, and $10^{\circ}$. The values of $\beta$ were taken to be $-5^{\circ},-2^{\circ}, 0.1^{\circ}$ and $5^{\circ}$, where the value of $\beta=0.1^{\circ}$ was used in the place of $\beta=0^{\circ}$ with the intent that any tendencies toward significant flow asymmetry might be identified by the computations.

The results for $\alpha=5^{\circ}$ are shown in figure 22 and highlight the good prediction of rolling moment, yawing moment, and side force for these conditions. The paper by Green ${ }^{25}$ also includes results at $\alpha=5^{\circ}$ and $10^{\circ}$ for $\mathrm{M}_{\infty}$ $=0.8$ and both of those plots show comparable agreement to what is shown here in figure 22. However, the story at this higher value of $\mathrm{M}_{\infty}=0.9$ becomes more complicated than the story for $\mathrm{M}_{\infty}=0.8$. Figure 23 illustrates some of the difficulty from the wind tunnel side of the story for a value of $\alpha=7^{\circ}$. Here the data are from both a NASA facility and an industrial tunnel and it is obvious that there are significant differences between two experimental data sets as conditions approach stall at this higher value of $\mathrm{M}_{\infty}$. Interestingly, the computational predictions, again done in the blind, fall between the two experimental curves.

The results for $\alpha=10^{\circ}$ are shown in figure 24. Here, for the first time, was a real break-down in the blind predictions, illustrated by the red symbols. Everything had been predicted so well by the computations up to this point that the research team was surprised that the data miss was so dramatic. However, Green ${ }^{25}$ was able to discern the reason for the mismatch by varying the initial conditions of the computation. For the original "blind" predictions, the initial conditions were just the free-stream conditions corresponding to the desired value of $\beta$. The later runs, conducted after seeing the discrepancies, assumed initial conditions corresponding to the converged solution for $\beta=0.1^{\circ}$.

The computations may have rediscovered what the experimental world has had to live with for many years. That is, when the flow can exhibit hysteresis, or multiple states, active steps have had to be taken in the wind tunnel to either quantify or minimize the hysteresis. Often, the solution to minimize hysteresis in the tunnel would be to start taking sideslip data from a nominal $\beta=0^{\circ}$ initial condition and then move in either the positive or negative $\beta$ directions. In this way, the flow states are going from less separation to more separation. This approach is similar to what Green has done here by using a nominally symmetric solution as the initial condition. More research is required here to determine if the computational differences are truly the result of hysteresis or not. Another approach for the CFD might be to run in a time-accurate mode and to see if that would solve, or capture, the mismatch for $\alpha=10 .^{\circ}$ The unsteady nature of the flow over the F/A-18E/F at these conditions is well documented. ${ }^{26,27}$

\section{Summary}

Under the CHSSI program and the CST portfolio, NAVAIR researchers have taken a fighter configuration, the pre-production F/A-18E/F, which is known to exhibit an abrupt wing stall nature, and have generally made blind predictions of both longitudinal and lateral/directional characteristics. In general, the TETRUSS code used has successfully captured longitudinal trends with horizontal tail increments and lateral/directional increments with sideslip for the undeflected horizontal tail case. The shortcomings identified during this investigation include (1) the apparently premature prediction of the wing stall when compared to the experimental wind tunnel data and (2) sensitivity to the initial conditions when computing sideslip values for $\mathrm{M}_{\infty}=0.9$. The premature prediction of wing stall may be due, in part, to the unsteady nature of the actual flow while the code is not being run in a timeaccurate mode. The significant impact of the initial conditions for the computations should not have been surprising because of the similar need to develop processes in the tunnels to control the impact of hysteresis.

\section{Boeing Commercial Airplanes}

Computational fluid dynamics has been used very successfully to develop the high-speed lines of Boeing's commercial transports since the 767. For attached flows, CFD has been able to predict the performance of a configuration with a great deal of confidence. This application, however, involves a very small part of the 
operational flight envelope of an airplane as can be seen in figure 25. There is a need to apply these CFD tools throughout the flight envelope because, as seen in figure 26, the larger amount of time currently spent during wind tunnel testing targets high-lift lines, loads, S\&C, and the flight simulator needs. While great reductions in tunnel time required for defining high-speed lines have been made, there has been very little corresponding progress in reducing the required time for the other needs. In order to achieve the goals of significant reductions in development costs and time to market, the use of CFD must be extended to the corners of the envelope, both flaps up and down.

\section{Transonic Pitch Characteristics}

Pitch characteristics for a tail-off transonic transport model are presented in figure 27. At first glance, the lift coefficient is nearly identical between CFL3D and wind tunnel test data for the range of $\alpha$ shown. However, a close inspection reveals that the CFD results do not fully capture non-linear $\mathrm{C}_{\mathrm{L} \alpha}$ effects in the mid- $\alpha$ region. Pitching moment comparisons reveal similar discrepancies between experimental data and CFD results. General trends with $\alpha$ are clearly captured, but CFD is not capturing all of the non-linear $\mathrm{C}_{\mathrm{M} \alpha}$ trends in the mid- $\alpha$ region. Comparisons at higher values of $\alpha$ reveal that CFD captures the general shape with a bit more instability than indicated by the tail-off test data. It is also worth noting that the offset between CFD and wind tunnel in the pitching moment curves is generally not of prime concern for commercial transport S\&C. The offset may be the result of experimental transition strips, model construction or residuals from mounting system corrections, and is typically corrected for by trimming with the stabilizer on a conventional commercial transport. It should be noted that while the agreement shown in figure 27 is much better than much that has been presented in the previous figures, there is always room for improvement.

\section{Spoiler Analysis}

Spoiler reversal CFD predictions are presented in figures 28 for a nacelles-off transonic transport. As detailed by Wilkinson, ${ }^{28}$ spoiler reversal is caused by adverse shock movement at higher values of $\alpha$. CFD predictions show that at low values of $\alpha$ the shock moves forward as the spoiler deploys, reducing lift and rolling the airplane as intended. At higher values of $\alpha$, however, results show the opposite trend, with the shock moving aft as the spoiler is deployed with a consequent increase in lift and adverse rolling moment. Experimental comparisons reveal the shock position from CFD is correctly predicted in each case. The importance of employing CFD for this case is that the CFD results aided in development of a theory explaining the spoiler reversal. Flow analysis revealed that at low values of $\alpha$ the flow past the stowed spoiler is subsonic, while at higher values of $\alpha$ the flow past the stowed spoiler is supersonic.

Subsequent spoiler analysis was extended to a typical transonic twinjet. Figure 29 illustrates slices of total pressure in the freestream, highlighting the vortices and wakes in the CFD simulation with outboard spoilers deployed (spoilers shown in red). The interaction of the wake and vortex system shedding from the wing on to the tail is of particular interest. These computations were performed with CFL3D and include a system of vortex generators as indicated in figure 30 and detailed by Tinoco. ${ }^{29}$ Figure 30 also shows near-surface streamlines in the vicinity of the deflected spoiler and a total pressure slice parallel to the leading edge illustrating the vortices shed by the vortex generators. Pressure distributions are compared to test data at inboard and outboard locations for one of the solutions in figure 31 . Comparisons reveal the solutions are relatively accurate with good correlation on the wing surface aft of the spoiler. The outboard location reveals a spike in the CFD data caused by the presence of a vortex generator. The distribution and density of pressure ports in the experimental data was inadequate to resolve this detail highlighted by the computation.

\section{Assessing the Effect of Reynolds Number on Longitudinal Control}

Reynolds number scaling effects on elevator control authority at a low Mach number are presented in figure 32 , and detailed by Tinoco ${ }^{29}$. CFD results show good correlation with experimental trends and a general increase in control authority with increasing Reynolds number out to flight conditions. Figure 33 illustrates that the flow physics behind the trend is the decreased flow separation aft of the hingeline as indicated by the reduction in red streamlines propagating from the trailing edge as Reynold number increases.

\section{Directional Stability}

Transonic directional stability characteristics were analyzed on a typical twinjet in sideslip using an unstructured grid and the CFD++ Navier-Stokes $\operatorname{code}^{30}$ with the $\mathrm{k}-\varepsilon-\mathrm{R}_{\mathrm{t}}$ turbulence model. ${ }^{31}$ Surface pressure 
distributions are shown in figure 34 for a transonic condition at positive $\beta$. Shock position differences (denoted by the red/green boundary) between port and starboard wings detail the shock movement induced by the sideslip. On the starboard wing, the shock position is strengthened and moves aft as the sideslip reduces the effective wing sweep for this largely attached flow condition. On the port wing the wing sweep is effectively increased, and the shock strength is reduced and moves forward.

Directional stability $\left(\mathrm{C}_{\mathrm{N} \beta}\right)$ comparisons with test data are shown in figure 35 . The figure supports 2 primary conclusions. First, CFD calculations under predict the level of directional stability. Second, the $\mathrm{M}_{\infty}$ trend is predicted relatively well out to $\mathrm{M}_{\infty}=0.94$. Longitudinal CFD calculations with the same grid provide essential insight towards understanding the level shift between the two curves. The lift curve slope $\left(\mathrm{C}_{\mathrm{L} \alpha}\right)$ is a little higher than experimental data. This causes the wing/body contribution to directional stability to be a little higher as well. Since the wing/body contribution to directional stability is negative, the CFD results end up a little lower than experimental data. Figure 36 shows conclusively that the discrepancy in $\mathrm{C}_{\mathrm{N} \beta}$ is in the wing/body term by showing the fin increment matches wind tunnel data quite well. The fin contribution is obtained by calculating $\left(\Delta \mathrm{C}_{\mathrm{N} \beta}\right)_{\mathrm{FIN}}=$ $\left(\mathrm{C}_{\mathrm{N} \beta}\right)^{\text {Fin On }}-\left(\mathrm{C}_{\mathrm{N}_{\beta}}\right)^{\text {Fin Off }}$ for both CFD and test data. Results show the fin increment is predicted accurately over a range of Mach numbers including near sonic conditions. This is particularly interesting since it shows CFD can provide accurate prediction well beyond the typical cruise condition for a conventional transport. It is also important to note that these were the first Navier-Stokes calculations performed in sideslip for this analysis and may not be grid converged or utilize the most appropriate turbulence model.

\section{Dynamic Derivatives}

Dynamic derivatives predicted by CFD calculations for rotational rate terms are also presented for 3 airplanes in figure 37. Results show consistent correlations between predictions using handbook methods for each of the configurations and the CFD calculations which were performed at a low transonic condition using the panel method A502 (PANAIR). Since static derivatives $\left(\mathrm{C}_{\mathrm{L}_{\alpha}}, \mathrm{C}_{\mathrm{N}_{\beta}}\right)$ from the panel code are consistently higher than handbook data, it is not surprising that dynamic derivatives are also higher. Consistency in the CFD to handbook differences allows use of the data even when the absolute levels are not precisely accurate.

\section{Summary}

By utilizing CFD, Boeing has already saved the company many dollars by reducing the time needed for wind tunnel testing. The company is now aggressively attempting to push the application of high-end CFD tools into the $\mathrm{S} \& \mathrm{C}$ challenge areas. The current examples describe the use of CFD to provide a better understanding of unexpected results from wind tunnel testing, as was the case for the spoiler reversal. High-end CFD, while too expensive to employ to populate entire data bases, can provide a level of detail in describing pressures over wings that can not be matched by conventional pressure measurement techniques in the tunnel. CFD has also shown the ability to predict Reynolds number trends on the horizontal stabilizer at low Mach numbers and has demonstrated the ability to predict the effect of $\mathrm{M}_{\infty}$ on directional stability. Finally, even employing lower order CFD such as panel methods to predict dynamic stability characteristics is shown to provide consistent increments from configuration to configuration.

\section{Air Force Research Laboratory (AFRL)}

The Computational Sciences Branch and Aerodynamic Configuration Branch of Air Vehicles Directorate, Air Force Research Laboratory (AFRL/VAAC and AFRL/VAAA, respectively), have a small effort underway to better understand the fundamental reasons CFD typically does a poor job of predicting S\&C. The goal is to determine what level of modeling fidelity is required to accurately predict stability quantities, i.e. what level of geometric fidelity, grid resolution, and turbulence modeling are necessary to get accurate results. The presumption is that the Navier-Stokes equations completely and correctly model this system, and it is just a matter of proper application of current ( $2^{\text {nd }}$ order) CFD tools that is necessary. The approach is to apply a very methodical analysis process to a relatively simple stability problem, addressing each of the areas in turn to determine best practices for applying CFD to S\&C problems. Therefore, a relatively simple geometry that has been tested experimentally, and yet which exhibits somewhat complex behavior in its stability characteristics, was 
considered. To simplify even further, initially only static pitching moment was considered, as even this most basic stability quantity has proven difficult to accurately predict with CFD.

The 1303 generic UCAV geometry was chosen because there is recent experimental data available at benign test conditions, it has a relatively simple delta wing planform, and it exhibits nonlinear behavior in pitching moment with angle of attack. ${ }^{32}$ There has also been some effort to ensure that the digital representation of the model is consistent with what was tested in the wind tunnel. The 1303 model was tested with various leading edge geometries, ranging from sharp to varying levels of roundness. Figure 38 shows the model setup during wind tunnel testing, while figure 39 indicates the pitch break at moderate angles of attack in the experimental data. Surface oil flow patterns, not shown, on the model indicated there is some type of leading edge rollup or vortex as the angle of attack increases, which likely results in the observed pitching moment behavior.

The sharp leading edge geometry was chosen for comparison, the intent being to eliminate possible issues with transition over a rounded leading edge. It is apparent from the model setup in the tunnel that the mounting sting and apparatus is large relative to the model. For that reason the initial geometry considered with CFD includes a simplified representation of the sting assembly (see figure 40). The portion of the sting close to the model is an accurate representation of the tunnel apparatus, but after a certain distance the sting cross section is held constant and extended to the edge of the computational domain. No other tunnel geometry was considered, so each of the following cases was run with freestream conditions at the farfield boundary. A number of increasingly refined unstructured meshes were created with VGRIDns, with cell counts of $566 \mathrm{~K}, 1.7 \mathrm{M}, 4.7 \mathrm{M}$, and $8.3 \mathrm{M}$ cells (see figure 41 ). The farfield boundary was a box approximately 17 body lengths away from the UCAV in each direction; symmetry conditions were assumed to reduce the grid sizes. The smallest grid was an Euler tetrahedral grid, while the others were viscous grids with prismatic cells close to the body. Anisotropic stretching of 25 to 1 was used for the cells at the leading and trailing edges of the model. The larger grids increased the grid density primarily about the leading and trailing edges of the model. The computations were run to match the test conditions of a Mach number of 0.25 , and Reynolds number of 4.28 million. For all viscous grids this resulted in an average $\mathrm{y}^{+}$of 0.5. All computations were run with the Air Vehicles Unstructured Solver (AVUS), formerly known as Cobalt $60{ }^{11}$. The viscous cases were run fully turbulent with either the Spalart-Allmaras one-equation turbulence ${ }^{33}$ model or Menter's SST two-equation turbulence model. ${ }^{34}$

Figure 42 compares the computed drag polar to the corrected wind tunnel data. As expected, the inviscid solution is the farthest off the data, but it still does a reasonable job predicting the correct character of the solution. At the lower values of drag both turbulence models do a reasonable job on both grids. The turbulent results on the $1.7 \mathrm{M}$ cell grid depart noticeably from the experimental data at the higher drag values, while the $4.7 \mathrm{M}$ cell grid tracks the data well. The choice of turbulence model has little impact on the results for this larger grid. So, as expected, CFD predictions of lift and drag when the flow is predominantly attached are generally quite good.

Figure 43 compares the computed pitching moment coefficient to both the corrected and uncorrected wind tunnel data. Since the computations do not include the tunnel walls, the expectation is that the corrected tunnel data would be best for comparison. As expected the inviscid results are quite far off, but still reproduce the character of the data to a relatively high angle of attack. The viscous results on the $1.7 \mathrm{M}$ cell grid mimic the inviscid results, indicating the grid does not have the necessary resolution to accurately predict the viscous character of the flow (regardless of turbulence model). The $4.7 \mathrm{M}$ cell grid corrects this, and for lower angles of attack tracks the corrected tunnel data fairly well. The turbulence models show slight differences. At the pitch break the computed results depart somewhat from the data, and maintain a somewhat constant offset from the data through the last computed angle of attack. Finally, one data point was computed on the largest $8.3 \mathrm{M}$ cell grid slightly after the pitch break, and shows a noticeable improvement relative to the $4.7 \mathrm{M}$ cell grid. Thus, the indication is that a sufficiently resolved grid would match the experimental data. But, an $8+\mathrm{M}$ cell grid for such a simple planform is much larger than experience with lift and drag coefficients would suggest.

Figure 44 shows computed surface oil flow patterns and pressure contours for two values of angle of attack $\left(4^{\circ}\right.$ and $\left.8^{\circ}\right)$ on the $4.7 \mathrm{M}$ cell grid with Menter's SST turbulence model. The solution at the higher angle of attack clearly indicates an attachment line for a leading edge roll-up/vortex, indicating the solution has captured the character of the experimental results. Figures 45 and 46 show comparisons of computed pressure coefficient with experiment at the 18-inch span location for these two angles of attack (semi-span is 30 inches). Surprisingly, at the lower angle of attack a major difference between the turbulence models is apparent, with Spalart-Allmaras 
showing much better agreement with the data on the least resolved grid. One would assume that this agreement is fortuitous. The higher angle of attack shows better agreement between both turbulence models and the data, though the two models under- or over-predict the data in different locations. In any case, the pressure coefficient comparisons show that even though the integrated quantity agreement improved with higher grid resolution, the discreet data agreement does not necessarily improve everywhere.

The next step in the process is to apply solution adaptation techniques to refine the grid only in the regions that need it. This appears to be the only way to obtain the accuracy that is needed, while (hopefully) keeping the grid size manageable. It may be necessary to add further geometric fidelity, such as the tunnel walls and sting assembly. As the flow separates at higher angles of attack, turbulence model differences will likely become more pronounced. This may then require that more advanced turbulence modeling techniques, such as detached eddy simulation (DES), be applied.

\section{Summary}

The effort is aimed at examining the grid requirements and turbulence model needs to achieve accurate results. This UCAV configuration has been chosen for this study because of its simplicity. It has been demonstrated that achieving convergence with more grid resolution in an integrated sense of forces and moments do not insure, as is well known, that individual pressure distributions are also converging.

\section{Acknowledgement}

The AFRL authors would like to acknowledge (1) the assistance of DoD HPCMP and the ASC MSRC for providing the computational resources that were used for this study and (2) The Technical Cooperation Program (TTCP) for its role in providing the experimental data, which was taken by QinetiQ with funding from the Ministry of Defence of the United Kingdom.

\section{Summary}

This paper documents the status of the efforts at four organizations to aggressively apply high-end CFD codes to the real-world problems and challenges associated with the prediction of the stability and control (S\&C) characteristics of a new flight vehicle. The informal working relationship between NASA Langley, Naval Air Systems Command (NAVAIR), Boeing Commercial Airplanes (BCA), and the Air Force Research Laboratory (AFRL) has been helpful in general and, particularly, because it allows all four organizations to have insights into the issues involving the range of vehicles being studied by these groups. These vehicles include, respectively, the Blended Wing Body (BWB), the F/A-18E Super Hornet, commercial transport configurations, and a notional Uninhabited Combat Air Vehicle, or UCAV. Lessons learned can be summarized for each organization.

The work by NASA Langley involved both static characteristics as well as ground-breaking efforts in the evaluation of BWB pitch damping. While the work is still underway, it is clear that further improvements are needed before consistent prediction of static longitudinal stability for the low- $\alpha$ region are possible. Impacts of wind-tunnel blade and post supports are predicted by the codes and appear to do a good job of estimating the offsets. In the area of pitch damping, it is found that RANS codes do a credible job of predicting pitch damping trends for lower values of $\alpha$ but may be missing the reduction in pitch damping measured in the tunnel near $\alpha=$ $24^{\circ}$. DES, which may be better equipped to track the complex separated flows through less dissipation, may be showing some advantage for that condition. Again, further work is needed in this area.

One of the objectives of the work at NAVAIR with the F/A-18E has been to address the concern of the traditional, experimentally driven S\&C community that many of the computational predictions have been made in the past with the CFD analysts already knowing the answer. Consequently, the NAVAIR work was carefully executed to make sure that the researcher did not know the answer before conducting the grid convergence studies or choosing any other running options. The "blind" longitudinal results represented trends in lift and pitching moment satisfactorily but disagreed with wind tunnel data for angle of attack at which wing stall occurred. The lateral "blind" results shown were particularly good for predicting rolling moment as a function of sideslip for a Mach number of 0.8 . The results at a Mach number of 0.9 were mixed with good agreement at lower values of angle of attack but completely missing the mark for an angle of attack of $10{ }^{\circ}$ Computations done after the initial 
mismatch, and not "blind," demonstrated that the initial conditions for the flow solution are important and give a very representative answer if the initial condition is taken as a computational solution appropriate for no sideslip. In retrospect, this is similar to the wind tunnel, where processes have been developed to minimize hysteresis effects in sideslip.

The work at BCA represents the most applied usage of the high-end CFD because that organization has been engaged on applying the CFD to that company's real-world challenges to augment and complement information that they develop in the wind tunnel. From the examples included in this paper, it is clear that the use of CFD was extremely useful for the spoiler reversal challenge. The codes not only confirmed what was being seen in thewind tunnel but also gave the critical insight to explain the flow physics. High-end CFD has also been used to successfully capture both Reynolds number trends and Mach number trends. Consistency in correlation across multiple airplanes is critical. Even panel methods can be employed incrementally when discrepancies are consistent across multiple airplanes as shown in the linear range dynamic derivative example.

The effort at AFRL is focused on learning about grid resolution and turbulence modeling requirements necessary to achieve an accurate solution. This is work still in progress but has already reiterated that it is necessary to examine pressures as well as integrated forces and moments to insure that solutions are truly converging as grid refinement is taking place.

This paper has summarized the work within the four organizations represented. The work has not yet achieved its goals of validation of the computational tools for the $\mathrm{S} \& \mathrm{C}$ challenges but is continuing so that these valuable, complementary tools to the existing wind tunnel tools can be more routinely integrated into the aircraft development cycle. With the ability of CFD to simulate (1) flight Reynolds numbers, (2) flight Mach numbers, and (3) free-air conditions without tunnel supports or walls and to provide unprecedented flow diagnostics, the practical implementation of CFD in the $\mathrm{S} \& \mathrm{C}$ arena will lead to reduced development times and more optimal designs.

\section{References}

${ }^{1}$ Wolowicz, C. H., Bowman, J. S., Jr., and Gilbert, W. P., "Similitude Requirements and Scaling Relationships as Applied to Model Testing." NASA TP 1435, August 1979.

${ }^{2}$ Delsinger, P., "Moore's Law Extends and Expands for the Future," URL: http://www.intel.com/labs/eml/painterview.htm.

${ }^{3}$ Chambers, J. R. and Hall, R. M., "Historical Review of Uncommanded Lateral-Directional Motions at Transonic Conditions," Journal of Aircraft, Vol. 41, No. 3, May-June 2004, pp. 436-447.

${ }^{4}$ Ball, D. N., "The Role of Computational Fluid Dynamics for Stability and Control--Is it time?" Paper in "COMSAC: Computational Methods for Stability and Control," Compiled by C. M. Fremaux and R. M. Hall, NASA/CP-2004-213028/PT2, April 2004, pp. 692-717.

${ }^{5}$ Fremaux, C. M. and Hall, R. M., (compilers) "COMSAC: Computational Methods for Stability and Control," NASA/CP-2004-213028/PT1, April 2004.

${ }^{6}$ Fremaux, C. M. and Hall, R. M., (compilers) "COMSAC: Computational Methods for Stability and Control," NASA/CP-2004-213028/PT2, April 2004.

${ }^{7}$ Liebeck, R. H., "Design of the Blended-Wing-Body Subsonic Transport", Journal of Aircraft, Vol. 41, No. 1., pp. 10-25, 2004.

${ }^{8}$ Vicroy, D. D., Murri, D. G., and Grafton, S. B., "Low-speed, Large Angle Wind Tunnel Investigation of a Subsonic Blended-Wing-Body Tri-jet Configuration," NASA CDTM-10044, 2004.

${ }^{9}$ Campbell, R. L., Carter, M. B., Pendergraft, O. C., Jr., Friedman, D. M., and Serrano, L., "Design and Testing of a Blended Wing Body with Boundary Layer Ingestion Nacelles at High Reynolds Numbers", AIAA-20050459, January, 2005.

${ }^{10}$ Anderson, W. K., and Bonhaus, D. L., "An Implicit Upwind Algorithm for Computing Turbulent flows on Unstructured Grid." Computers and Fluids, Vol 23, No. 1, 1994, pp. 1-21.

${ }^{11}$ Strang, W. Z., Tomaro, R. F., and Grismer, M. J., "The Defining Methods of Cobalt ${ }_{60}$ : A Parallel, Implicit, Unstructured Euler/Navier-Stokes Flow Solver," AIAA 99-0786, January 1999.

${ }^{12}$ Abdol-Hamid, K. S., Massey, S. J., and Elmiligui, A. A., "PAB3D Code Manual". Cooperative development by the Configuration Aerodynamics Branch, NASA Langley Research Center and Analytical 
Services \& Materials, Inc. Hampton, VA.

${ }^{13}$ Spalart, P. R. and Allmaras, S. R., "A One-Equation Turbulence Model for Aerodynamic Flows," La Recherche Aerospatiale, No. 1, pp. 5-21, 1994.

${ }^{14}$ Girimaji, S. S., "Fully-Explicit and Self-Consistent Algebraic Reynolds Stress Model," Institute for Computer Applications in Science and Engineering, 95-82, December 1995.

${ }^{15}$ Pao, S. P., Biedron, R. T., Park, M. A., Fremaux, C. M., and Vicroy, D. D., "Navier-Stokes Computations of Longitudinal Forces and Moments for a Blended Wing Body," AIAA-2005-0045, January 2005.

${ }^{16}$ Frink, N.T., Pirzadeh, S. Z., Parikh, P. C., and Pandya, M. J., "The NASA Tetrahedral Unstructured Software System (TetrUSS)", The Aeronautical Journal, Vol. 104, No. 1040, October, 2000, pp 491 - 499.

${ }^{17}$ Biedron, R. T., Vatsa, V. N., and Atkins, H. L.: "Simulation of Unsteady Flows Using an Unstructured Navier-Stokes Solver on Moving and Stationary Grids", AIAA 2005-5093, June 2005.

${ }^{18}$ Forsythe, J., Hall, R., Morton, S.: "Calculations of Pitch Damping for the Blended Wing Body Using RANS and DES", Submitted for the $44^{\text {th }}$ AIAA Aerospace Sciences Meeting, Reno, NV. January 2006.

${ }^{19}$ Menter, F. R., "Improved Two-Equation k- $\omega$ Turbulence Model for Aerodynamic Flows," NASA TM $103975,1992$.

${ }^{20}$ Spalart, P. R., Jou W-H., Strelets M., and Allmaras, S. R., "Comments on the Feasibility of LES for Wings, and on a Hybrid RANS/LES Approach," Advances in DNS/LES, 1st AFORSR Int. Conf. on DNS/LES, Aug 4-8, 1997, Greyden Press, Columbus Ohio.

${ }^{21}$ Frink, N.T., Pirzadeh, S.Z., Parikh, P.C., and Pandya, M.J., "The NASA Tetrahedral Unstructured Software System (TetrUSS)", The Aeronautical Journal, Vol. 104, No. 1040, October, 2000, pp 491 - 499

${ }^{22}$ Pandya, M.J., Frink, N.T., Abdol-Hamid, K.S., and Chung, J.J., "Recent Enhancements to USM3D Unstructured Flow Solver for Unsteady Flows", AIAA Paper 2004-5201, 22 ${ }^{\text {nd }}$ Applied Aero Conference, August, 2004.

${ }^{23}$ Pandya, M. J., Frink, N. T., and Noack, R. W., "Progress Toward Overset-Grid Moving Body Capability in the USM3D Unstructured Flow Solver", AIAA Paper No. 2005-5118, June, 2005

${ }^{24}$ Hall, R. M., Woodson, S. H., and Chambers, J. R., "Overview of the Abrupt Wing Stall Program," Progress in Aerospace Sciences, Vol. 40, No. 7, October 2004, pp. 417-452.

${ }^{25}$ Green, B. E., and Chung, J. J., "CFD Predictions of the Stability and Control Characteristics of the PreProduction F/A-18E," AIAA Paper No. 2005-6122, August 2005.

${ }^{26}$ Schuster, D. M. and Byrd, J. E., "Transonic Unsteady Aerodynamics of the F/A-18E at Conditions Promoting Abrupt Wing Stall. Journal of Aircraft, Vol. 41, No. 3, May-June 2004, pp. 485-492.

${ }^{27}$ Forsythe, J. R. and Woodson, S. H., "Unsteady Computations of Abrupt Wing Stall Using Detached-Eddy Simulation. Journal of Aircraft, Vol. 42, No. 3, May-June 2005, pp. 606-616.

${ }^{28}$ Wilkinson, W.W., Lines, T.R., Yu, Neng J., "Navier-Stokes Calculations for Massively Separated Flows," AIAA Paper 96-2383, June 1996.

${ }^{29}$ Tinoco, E.N., Bogue, D.R., Kao, T.J., Li, P., Ball, D.N., "Progress Towards CFD For Full Flight Envelope," presented at the Royal Aeronautical Society (RAeS) Aerospace Aerodynamics Research Conference in London, England, held on September 14-15, 2004. Note: (The paper is also going to be published in The Aeronautical Journal as Paper 2962 at some future date unknown at this time.)

${ }^{30}$ O.Peroomian and S. Chakavarthy "A 'Grid-Transparent' Methodology for CFD," AIAA Paper No. 97-0724, January 1997.

${ }^{31}$ U.Goldberg, O.Peroomian, and S. Chakavarthy "Application of k-e-R Turbulence Model to Wall-bounded Compressive Flows," AIAA Paper No. 98-0323, January 1998.

${ }^{32}$ Bruce, R. J. et al.: "Low Speed Wind Tunnel Tests on the 1303 UCAV Concept", QinetiQ/FST/TR025502/1.0, March 2003. The 1303 UCAV work was performed under the auspices of TTCP AER TP-5. Data was obtained by QinetiQ and was funded by the UK MOD.

${ }^{33}$ Spalart, P. R. and Allmaras, S. R.: "A One-Equation Turbulence Model for Aerodynamic Flows", AIAA 920439, January 1992.

${ }^{34}$ Menter, F. R.: "Zonal Two Equation k- $\omega$ Turbulence Models for Aerodynamic Flows", AIAA 93-2906, July 1993. 
Figures

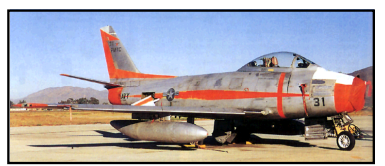

F-86

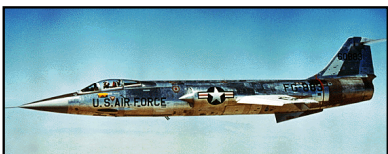

F-104

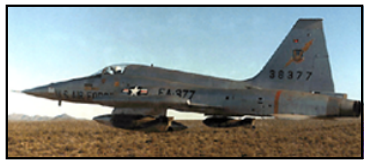

F-5

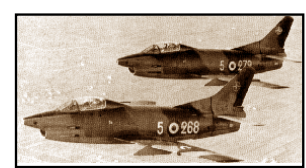

G-91

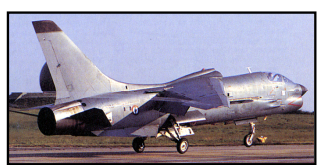

F-8

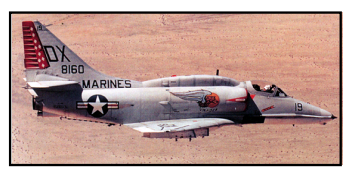

A-4

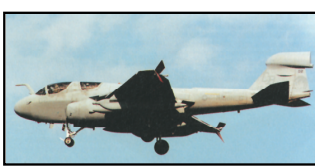

EA-6B

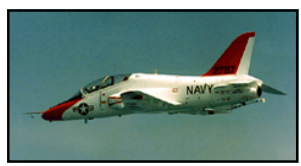

T-45

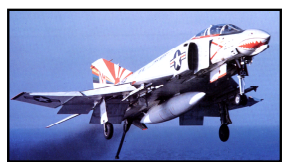

$\mathbf{F}-4$

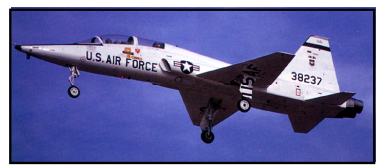

T-38

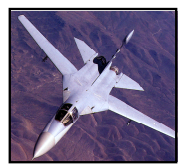

F-111

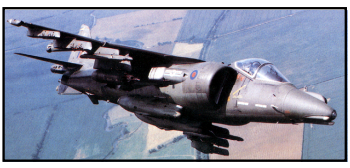

Harrier

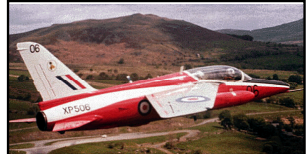

Gnat

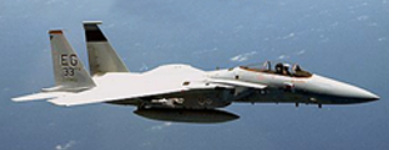

F-15

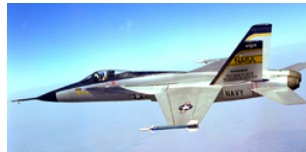

YF-17

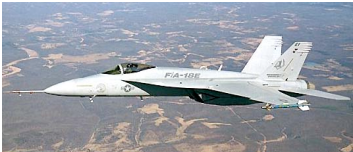

F/A-18E

Figure 1. Examples of high performance military aircraft that have experienced transonic, uncommanded lateral motions, such as wing rock or drop.

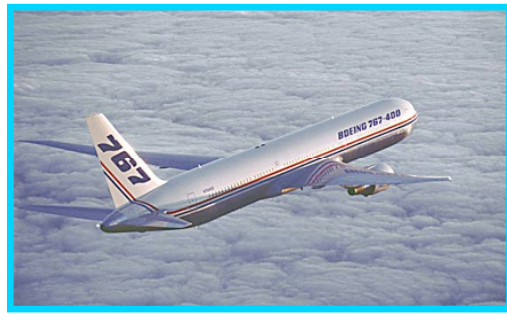

767 with raked wing extensions

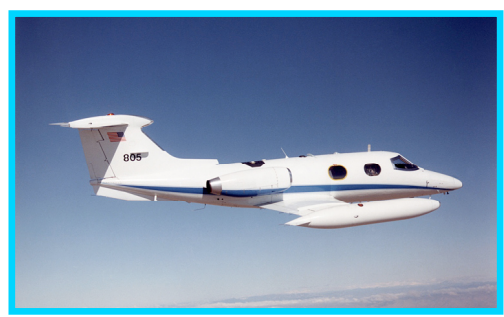

Lear 23

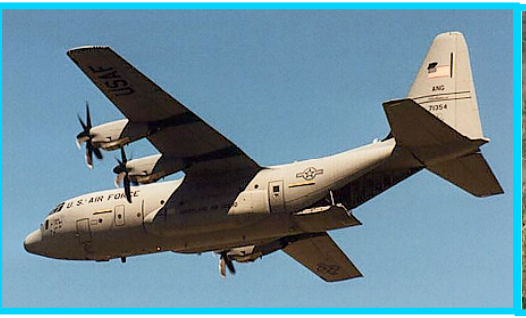

C-130 J

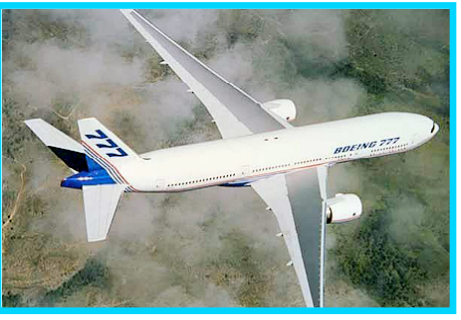

777

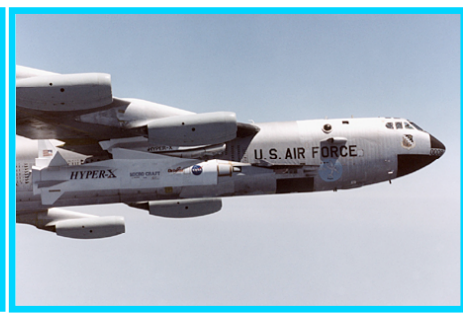

NASA X-43 Stack

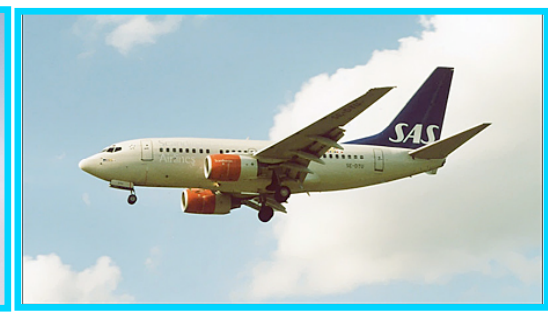

737 New Generation

Figure 2. Examples of other aircraft having unexpected stability and control characteristics in flight. 


\begin{tabular}{|l|l|}
\multicolumn{1}{c}{ CFD'ers } & \multicolumn{1}{c}{ S\&C'ers } \\
\hline Flow physics & Forces \& Moments \\
\hline Incipient separation & Massive separation \\
\hline Lift, Drag, L/D & 6 components \\
\hline Design point & Envelope \& beyond \\
\hline Symmetric flight & Alpha \& beta \\
\hline Static aircraft & 6 DOF motions \\
\hline $1 \%$ accuracy & Plus or minus \\
\hline Optimize & Cut \& try \\
\hline S\&C-challenged & CFD-challenged \\
\hline
\end{tabular}

Figure 3. Legacy differences between the CFD community and the S\&C community.

\subsection{3-scale BWB in 14- x 22-ft ST}
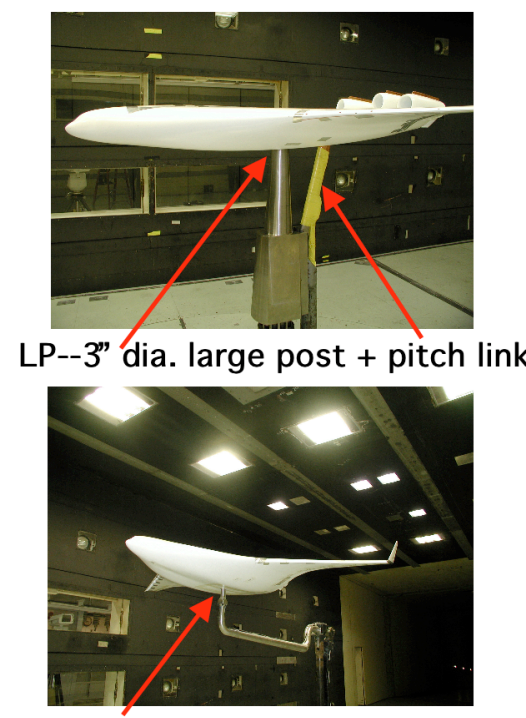

SP--1.2' dia. small post

\subsection{2-scale BWB in NTF}

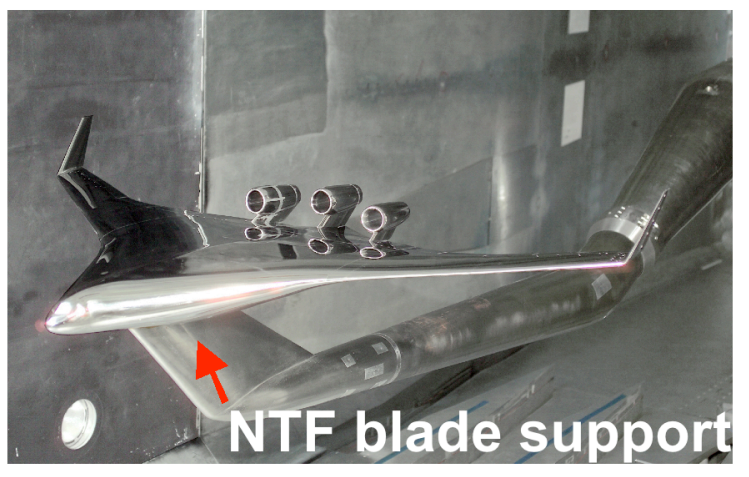

Figure 4. Various BWB model installations used during the experimental program. 


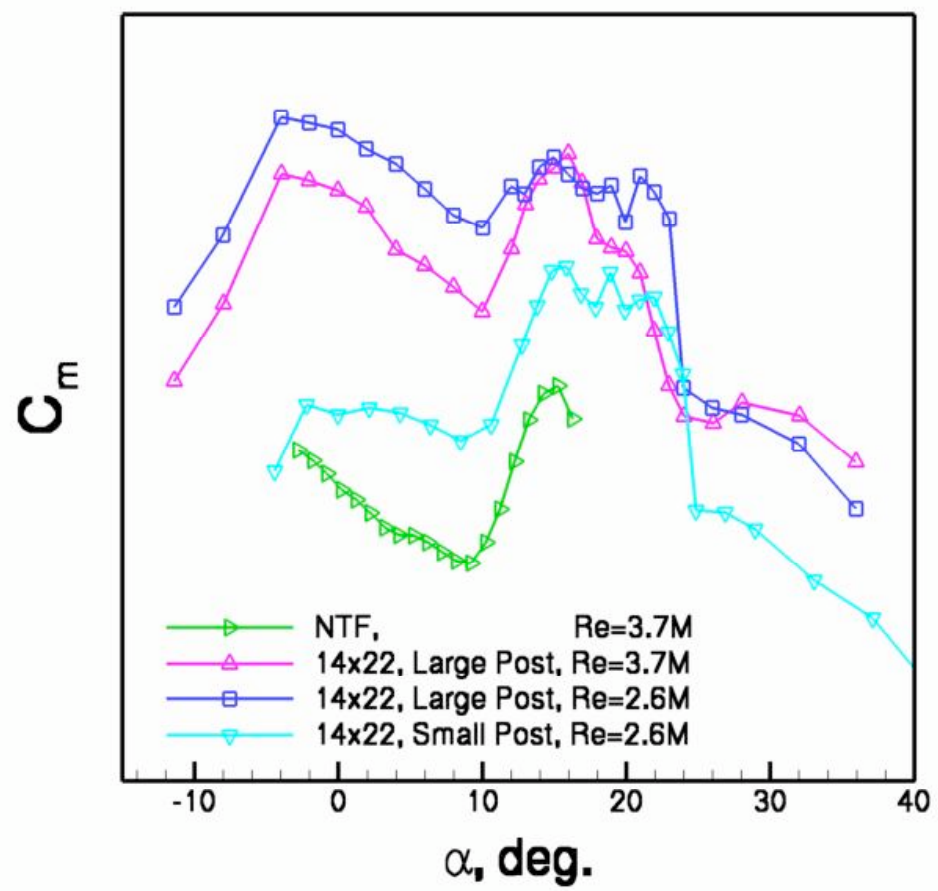

Figure 5. Comparison of experimental data sets for static BWB pitching moment; $\mathrm{M}_{\infty}=0.2$ (nominal).

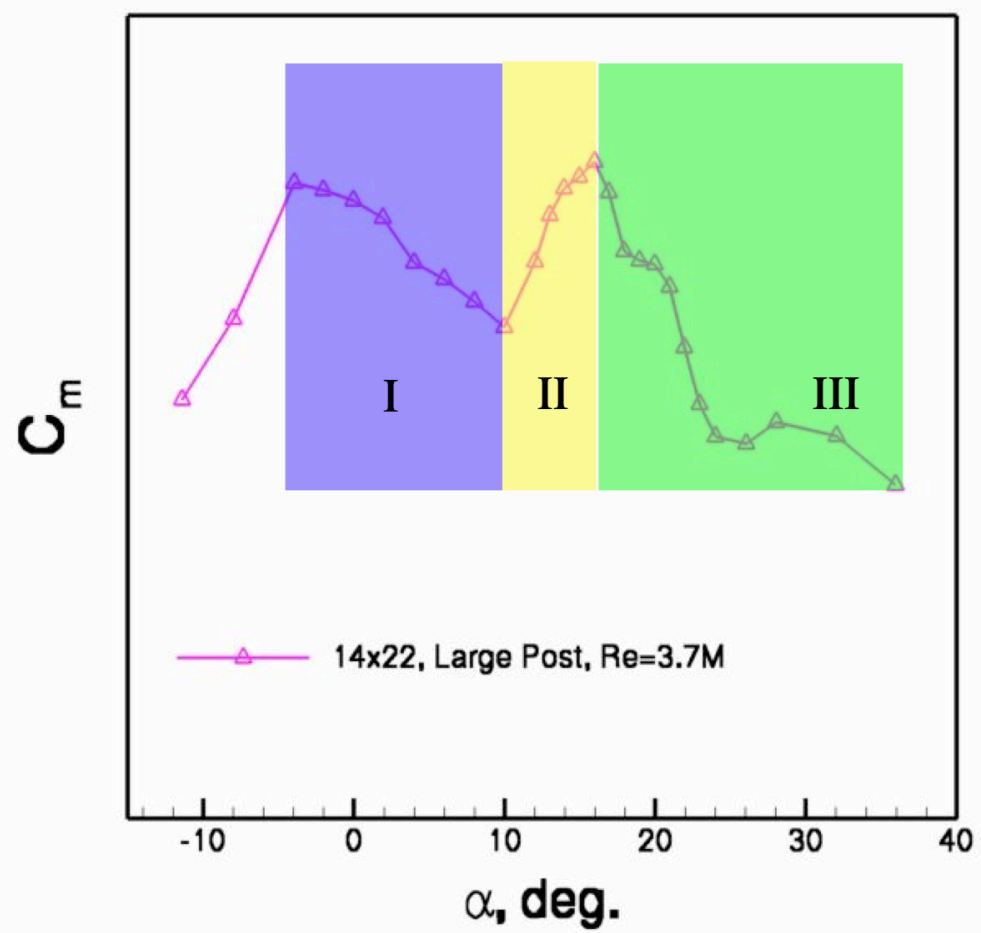

Figure 6. Key regions of interest for $\mathrm{S \& C}$. Region I is longitudinally stable and generally represents attached flow. Region II contains the unstable pitch break and continuing breakdown of flow. Region III represents a stable flow after stall. 


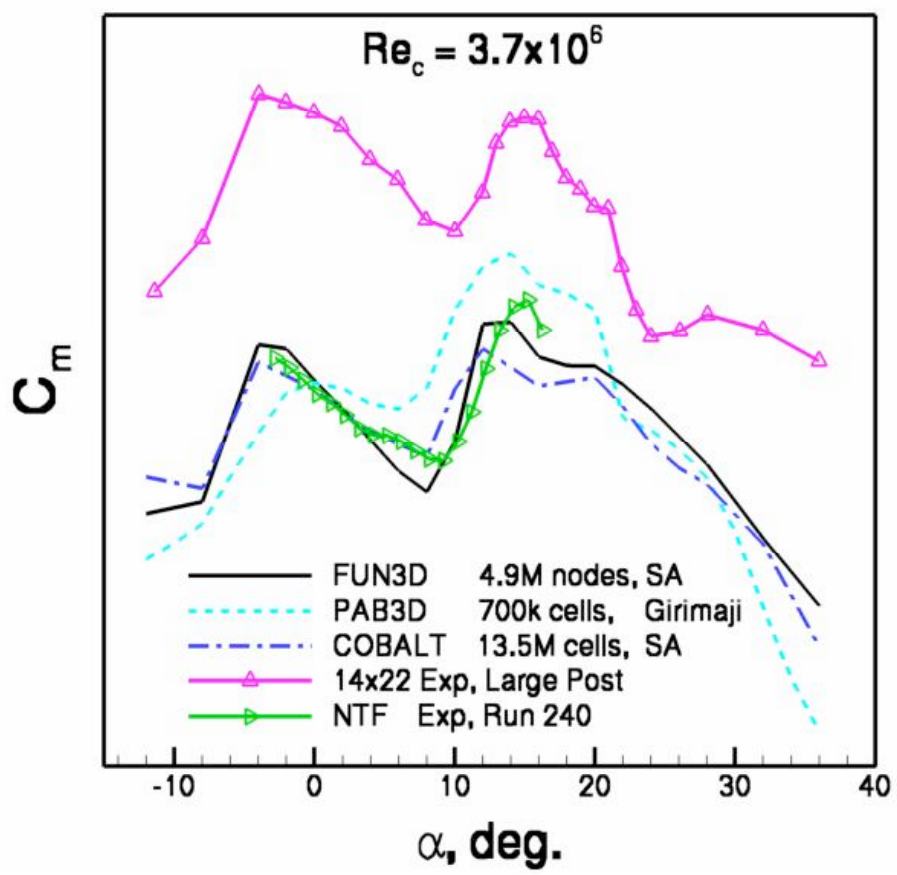

Figure 7. Comparison of computed static BWB pitching moment with experiment; $\operatorname{Re}_{\mathrm{c}}=3.7$ million, $\mathrm{M}_{\infty}=\mathbf{0 . 2}$.

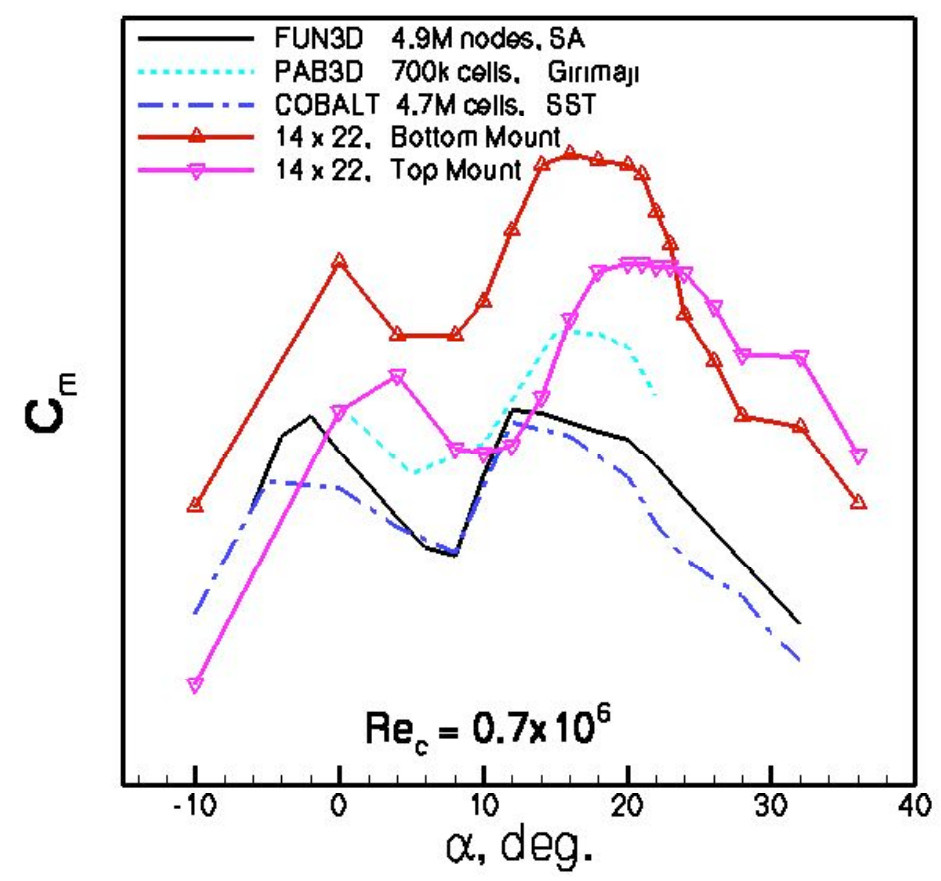

Figure 8. Comparison of computed static BWB pitching moment with experiment; $\mathrm{Re}_{\mathrm{c}}=0.7$ million, $\mathrm{M}_{\infty}=0.1$. 


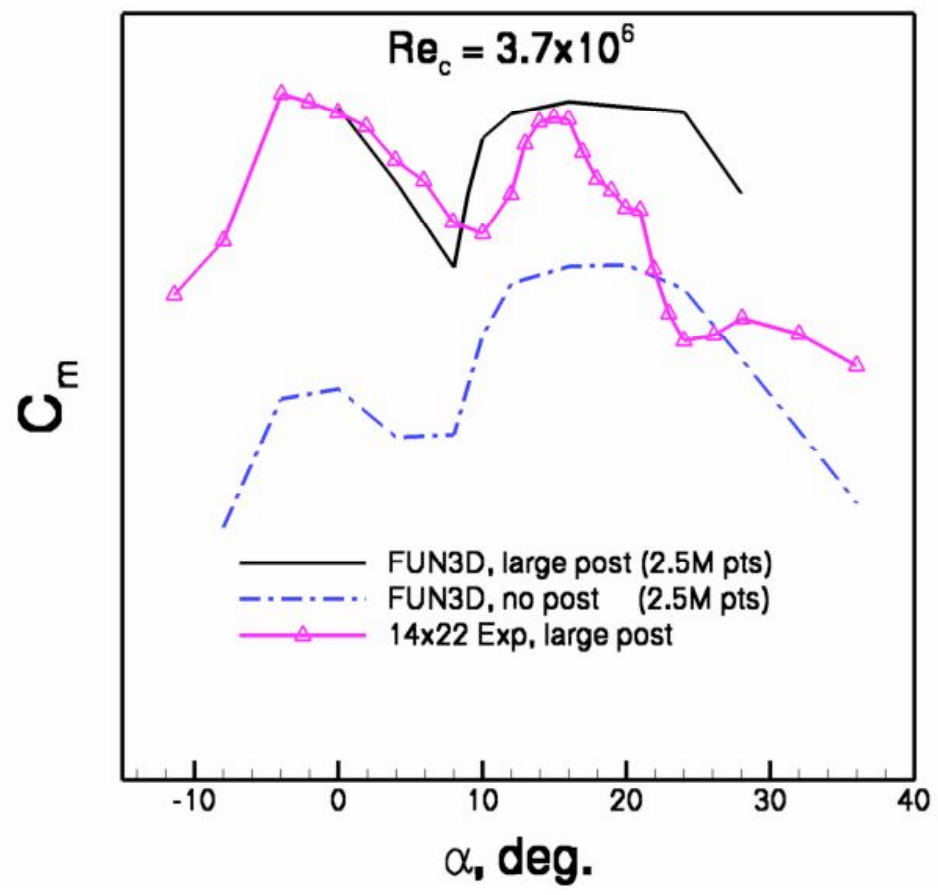

Figure 9. Effect of 14- x22-ft ST large post mount on computed static pitching moment for $\operatorname{Re}_{c}=3.7$ million, $\mathrm{M}_{\infty}=\mathbf{0 . 2}$.

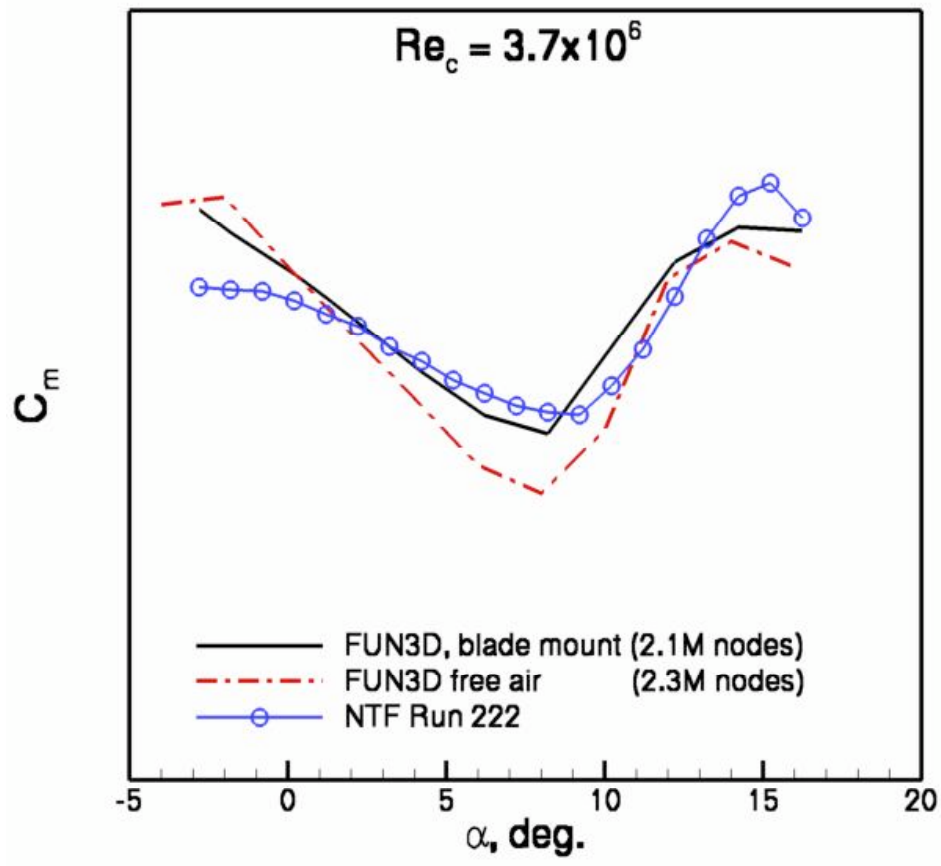

Figure 10. Effect of NTF blade mount on computed static pitching moment for $\operatorname{Re}_{c}=3.7$ million, $M_{\infty}=0.2$. 


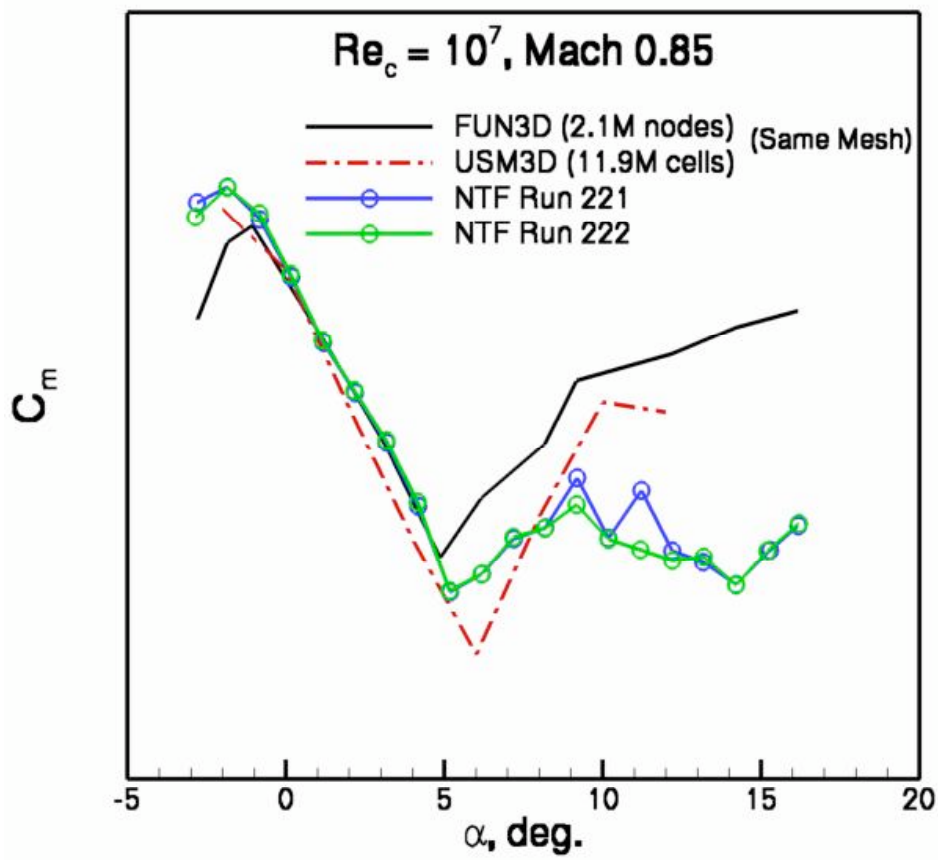

Figure 11. Comparison of computations including the blade support and experimental static pitching moment; $\operatorname{Re}_{\mathrm{c}}=\mathbf{1 0}$ million, $\mathrm{M}_{\infty}=\mathbf{0 . 8 5}$.

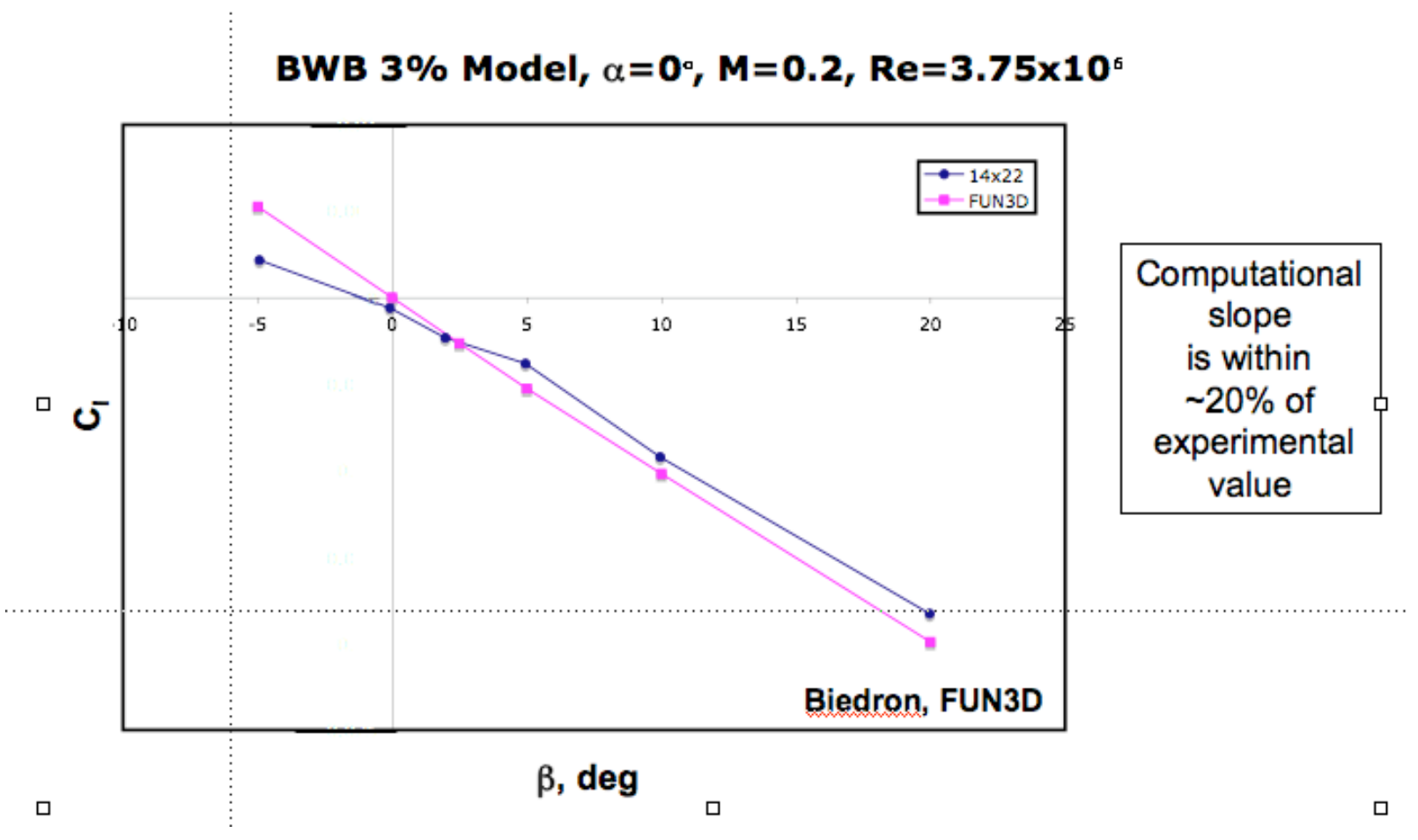

Figure 12. Comparison of computed and experimental rolling moment versus sideslip; $\alpha=0^{0}, \operatorname{Re}_{\mathrm{c}}=3.75$ million, $\mathrm{M}_{\infty}=\mathbf{0 . 2 0}$. 


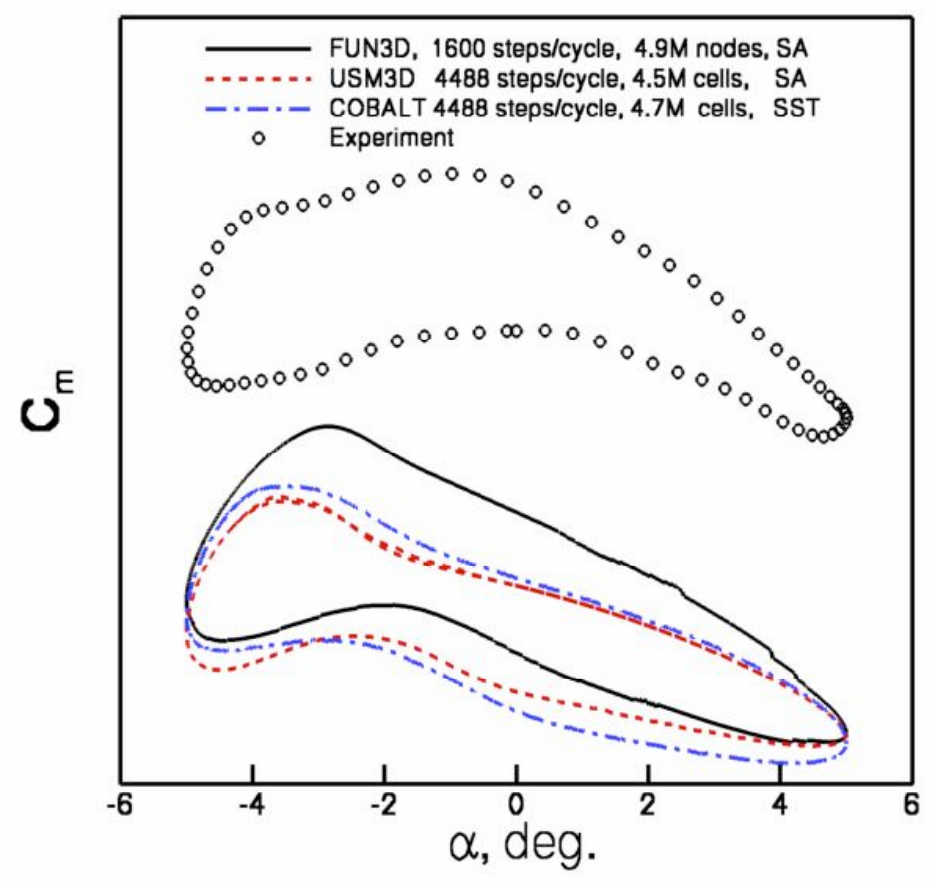

Figure 13. Dynamic pitching moment as a function of $\alpha$ for a forced oscillation of $+/-5$ degrees about $\alpha=0^{0}$. $\operatorname{Re}_{\mathrm{c}}=0.7$ million.

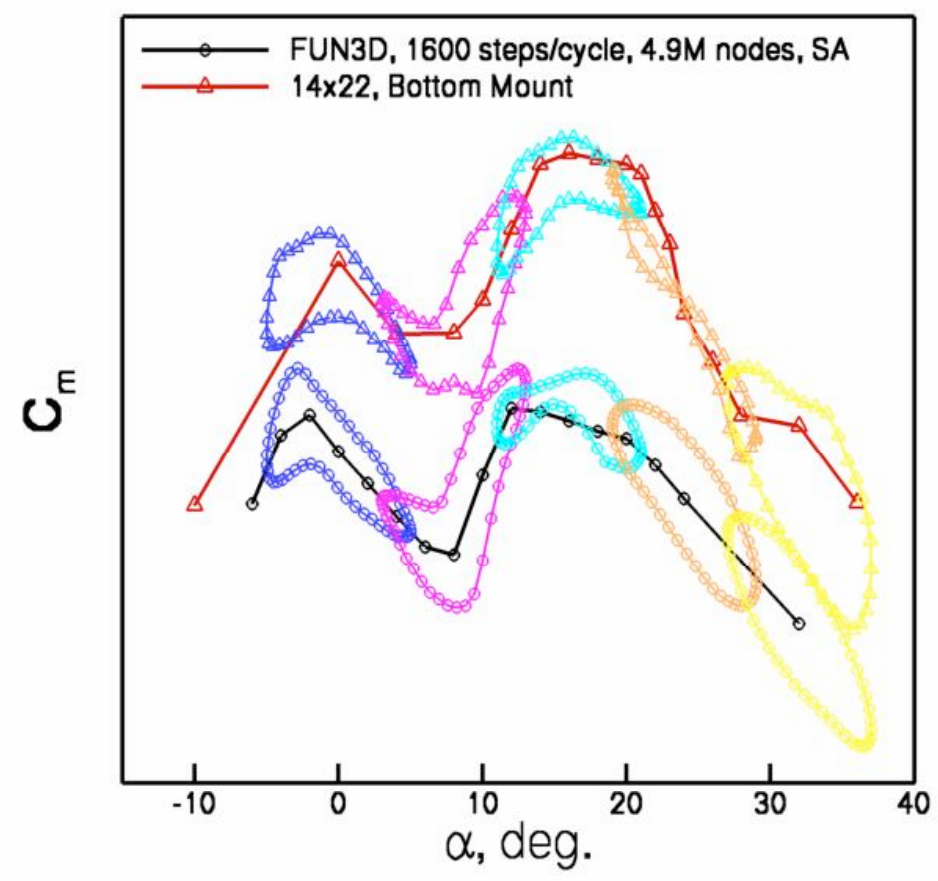

Figure 14. Dynamic and static pitching moments as a function of $\alpha$; forced oscillations of $+/-5$ degrees; $\operatorname{Re}_{c}=0.7$ million. 


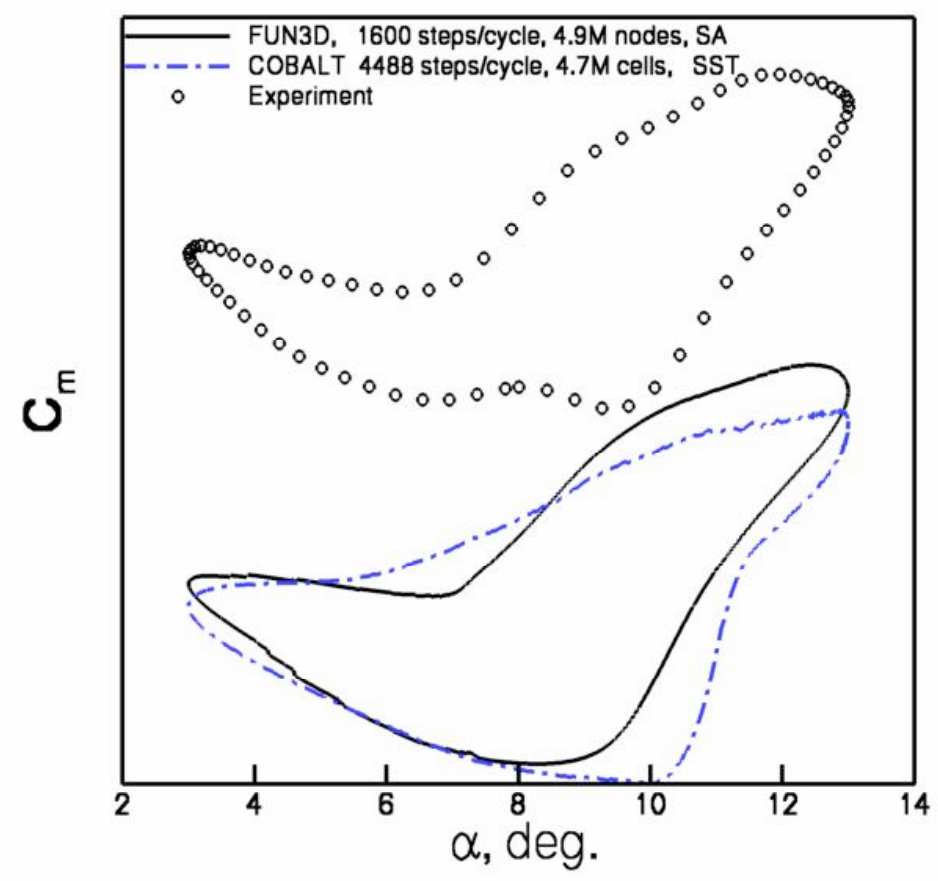

Figure 15. Dynamic pitching moment as a function of $\alpha$ for a forced oscillation of $+/-5$ degrees about $\alpha=8^{0}$. $\operatorname{Re}_{\mathrm{c}}=0.7$ million.

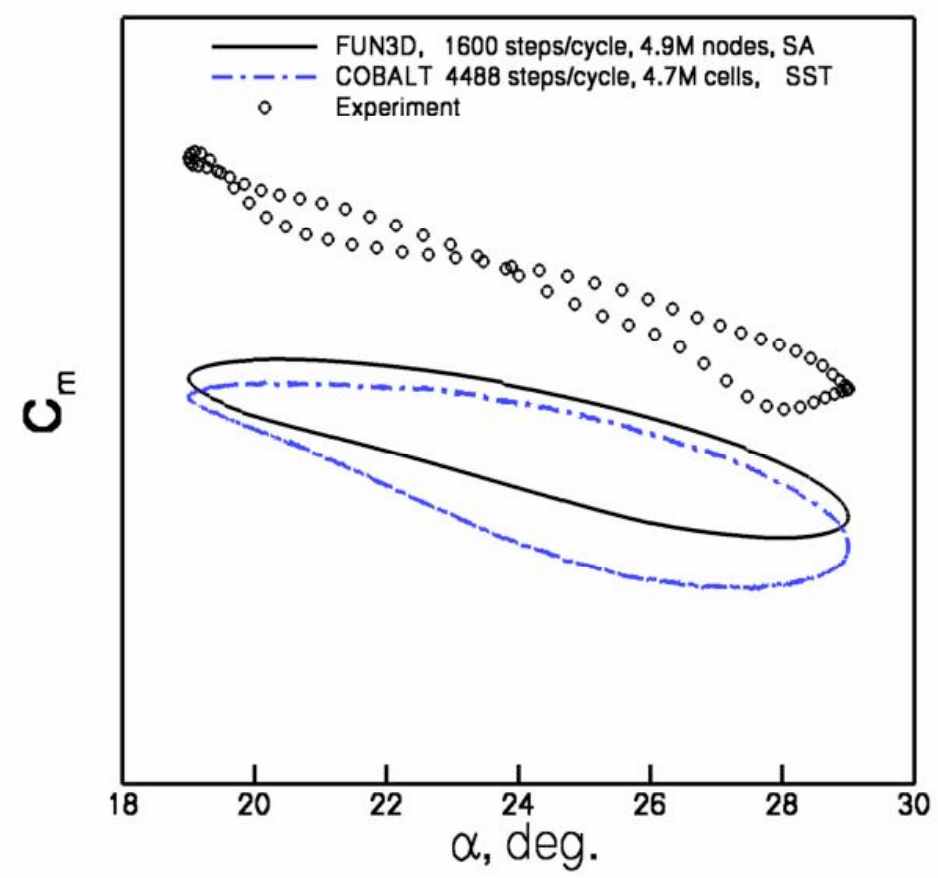

Figure 16. Dynamic pitching moment as a function of $\alpha$ for a forced oscillation of $+/-5$ degrees about $\alpha=24^{\circ}$. $\operatorname{Re}_{\mathrm{c}}=0.7$ million. 


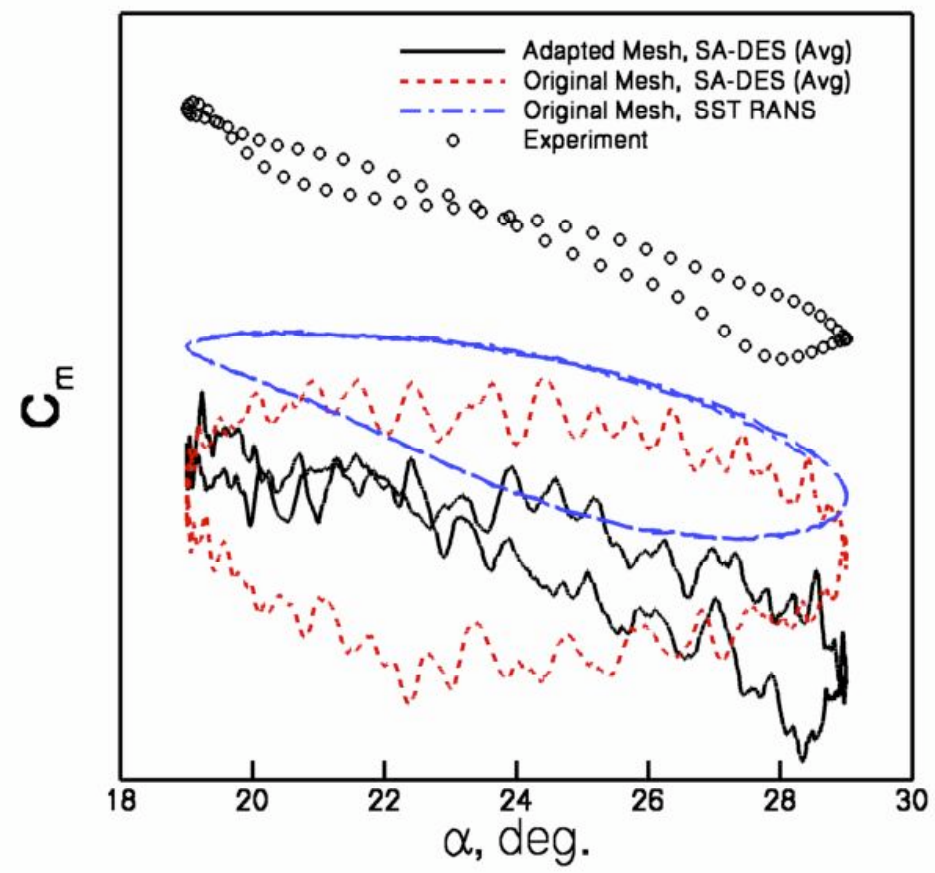

Figure 17. DES and RANS simulations from COBALT for oscillations of +/- 5 degrees about $\alpha=24^{\circ}$. $\operatorname{Re}_{\mathrm{c}}=\mathbf{0 . 7}$ million.

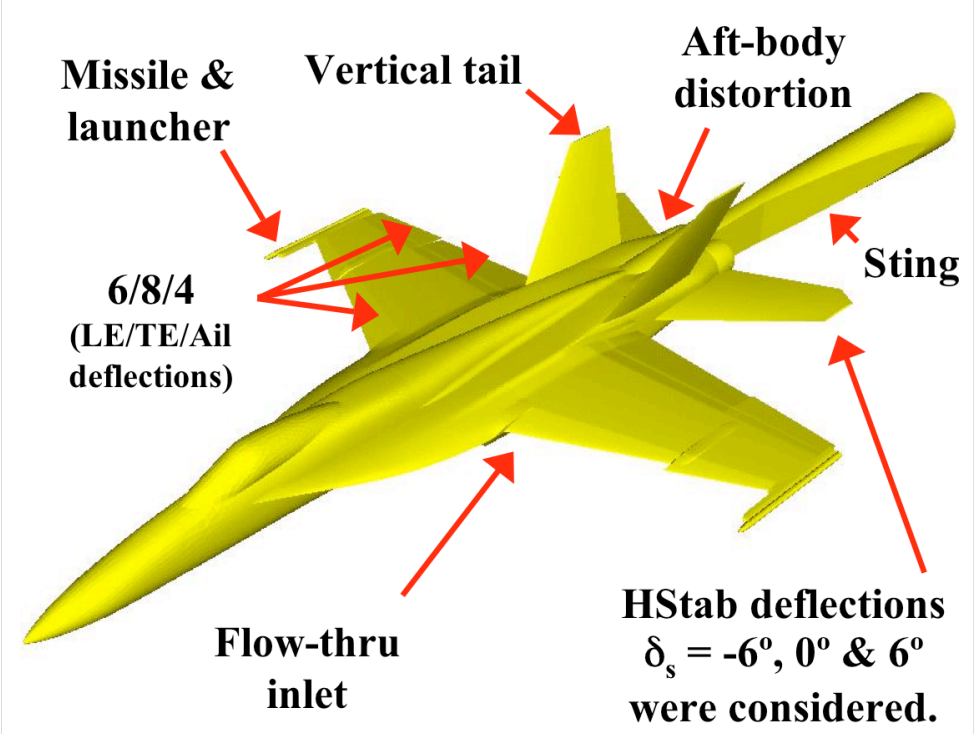

Figure 18. Computational geometry of the 0.08-scale wind tunnel model of the pre-production F/A-18E Super Hornet. 


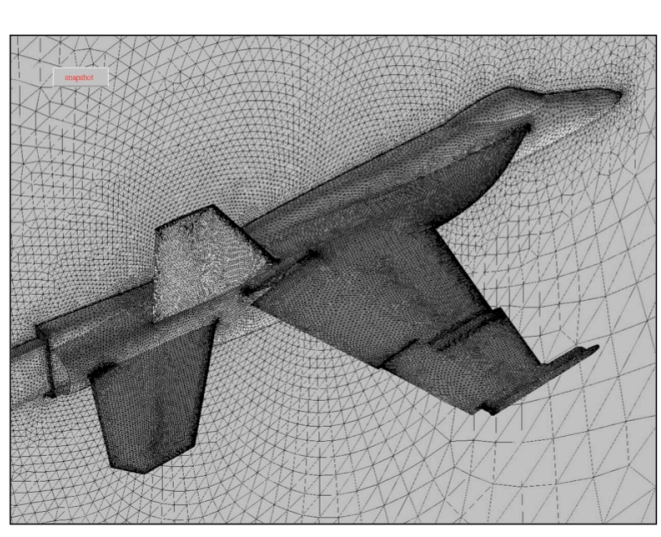

Medium Grid w/ Tails

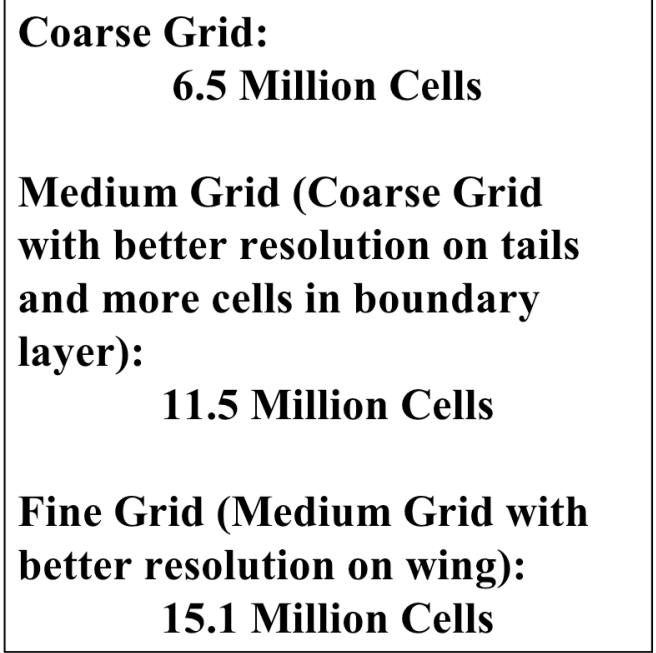

Figure 19. Grids based on F/A-18E wind tunnel model geometry with $6^{0} / 8^{0} / 4^{0}$ flaps.

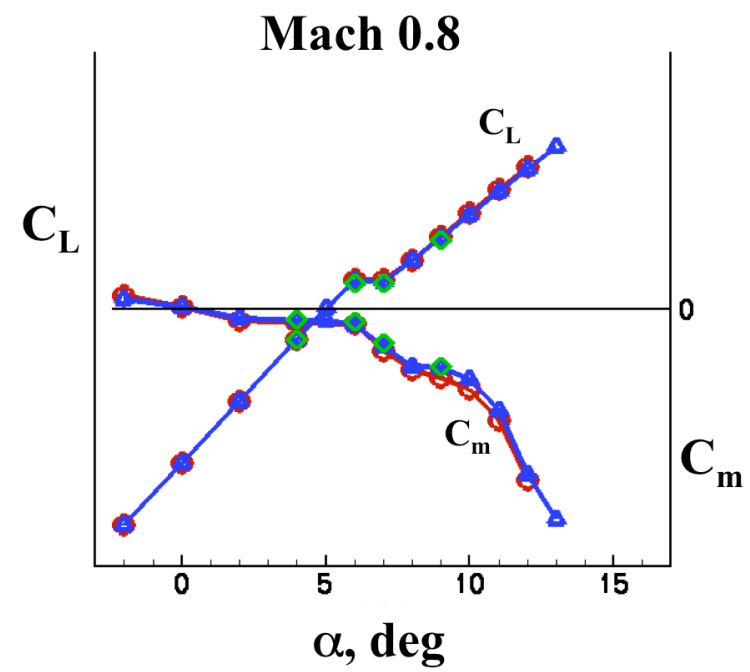

\section{Coarse Grid}

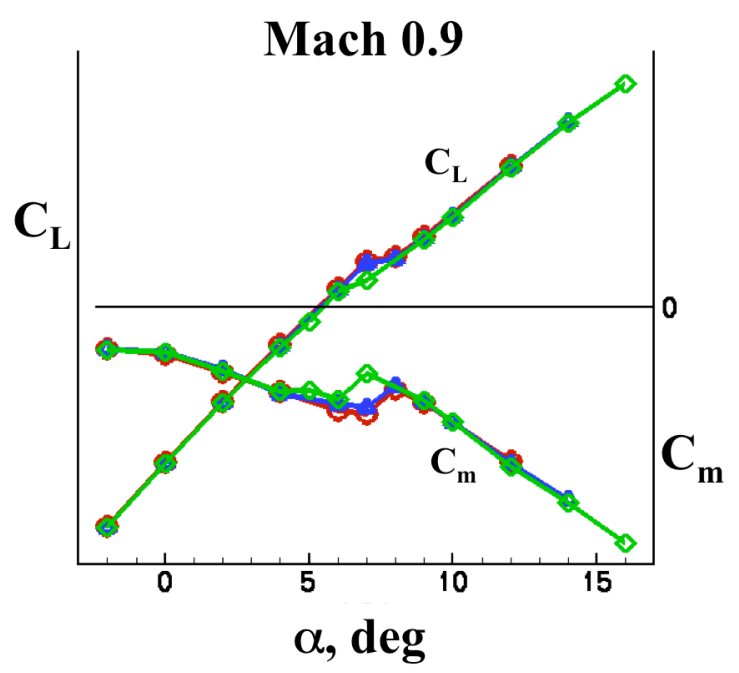

Medium Grid Fine Grid

Figure 20. Grid refinement study. 


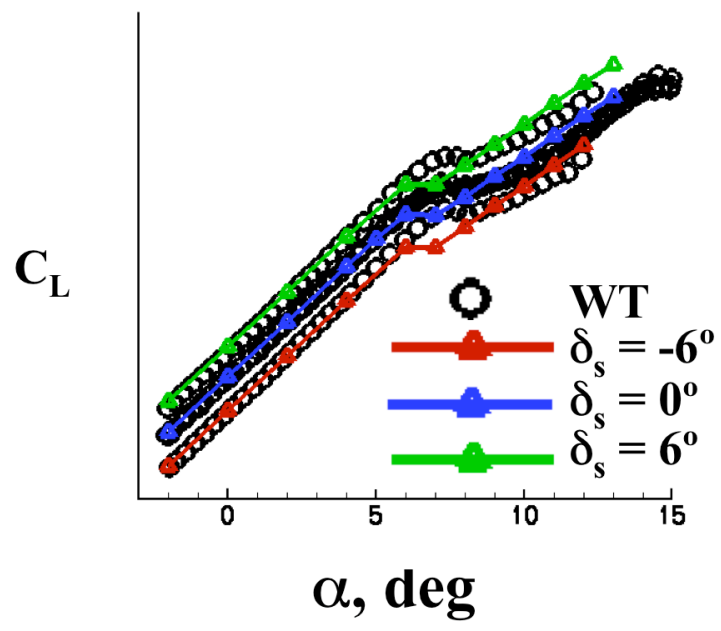

- Wind Tunnel Data

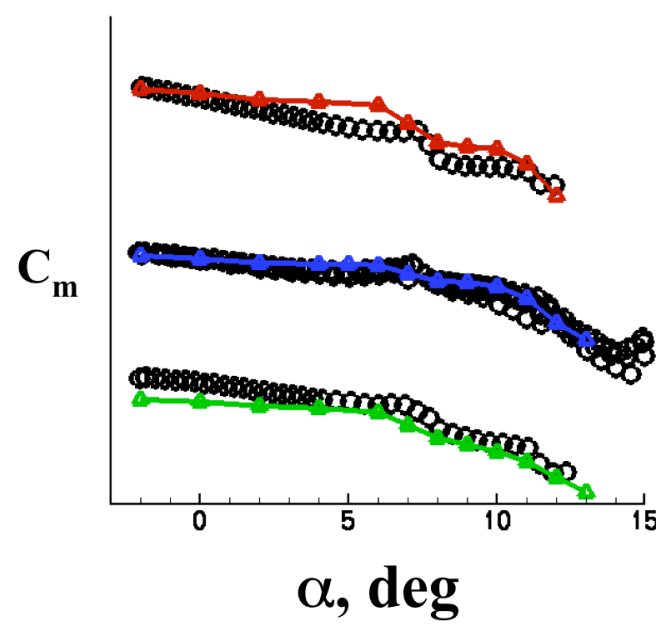

USM3Dns (medium grid

Figure 21. Lift and pitching moment for 3 horizontal tail deflections. $M_{\infty}=0.8, \operatorname{Re}_{c}=3.9$ million.
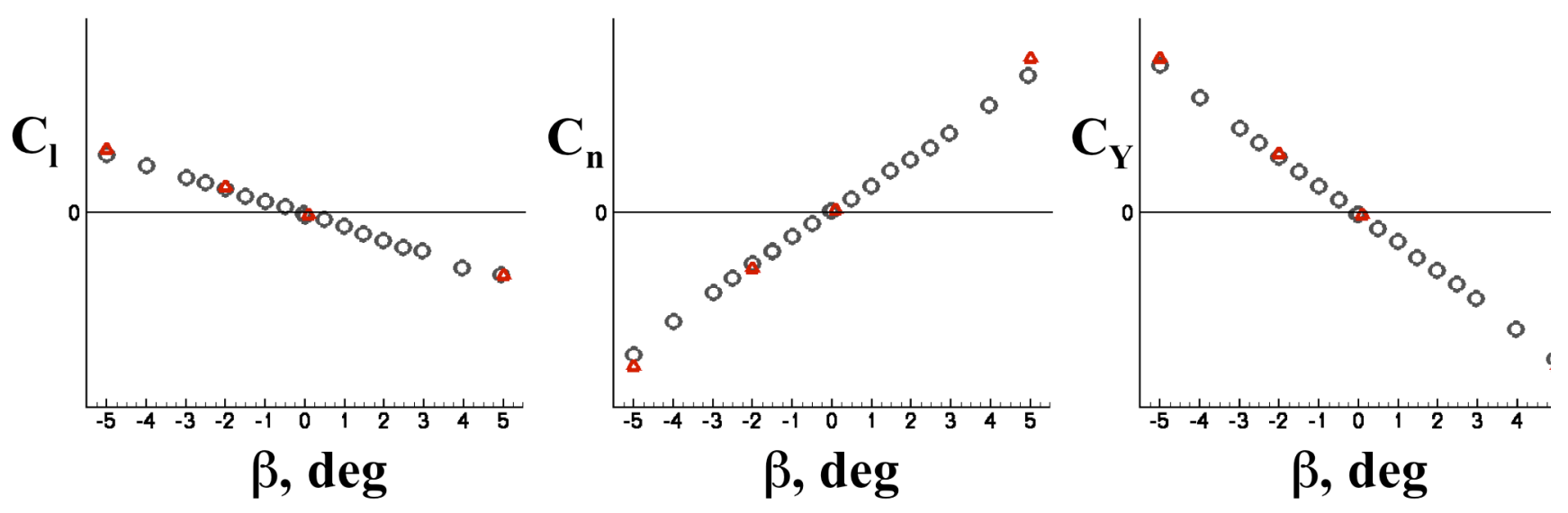

- Wind Tunnel Data

$\Delta$ USM3Dns

Figure 22. F/A-18E in sideslip, $M_{\infty}=0.9, \operatorname{Re}_{c}=4.0$ million, $\alpha=5^{\circ}$. 


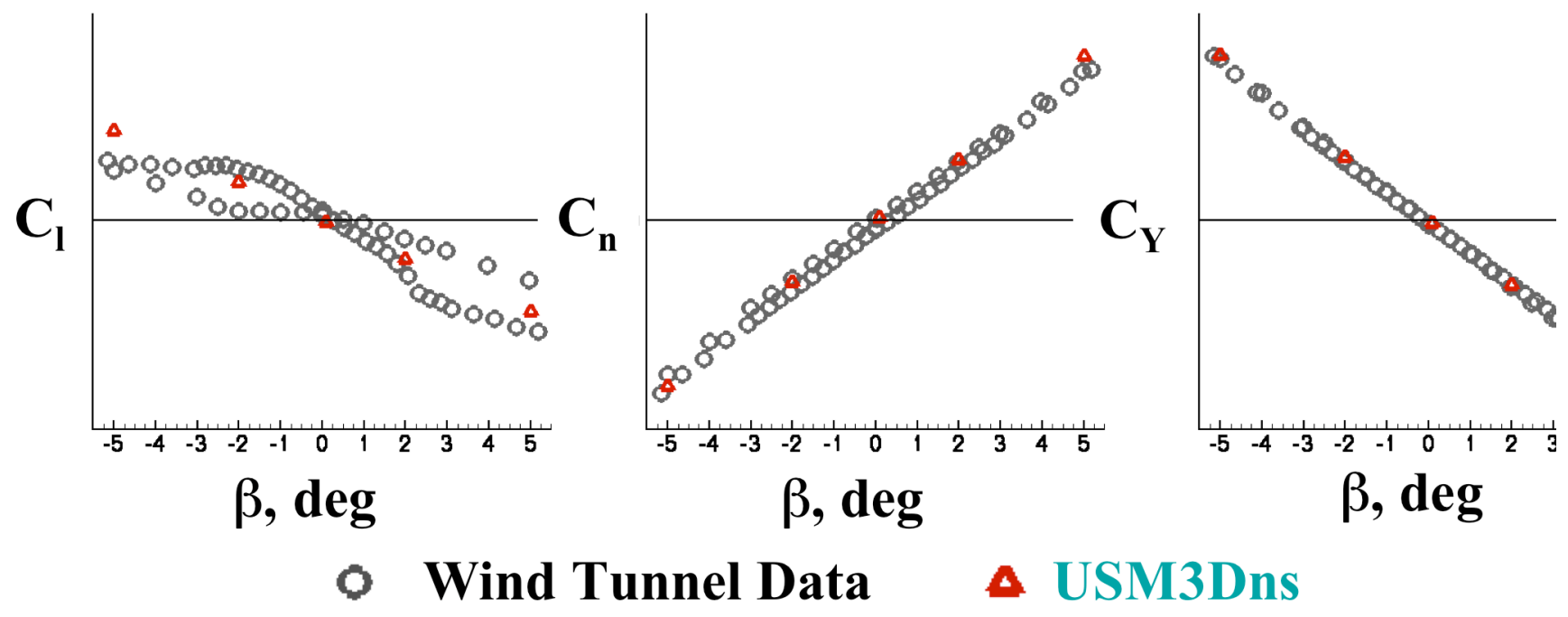

Figure 23. F/A-18E in sideslip, $M_{\infty}=0.9, \operatorname{Re}_{\mathrm{c}}=4.0$ million, $\alpha=7^{0}$.

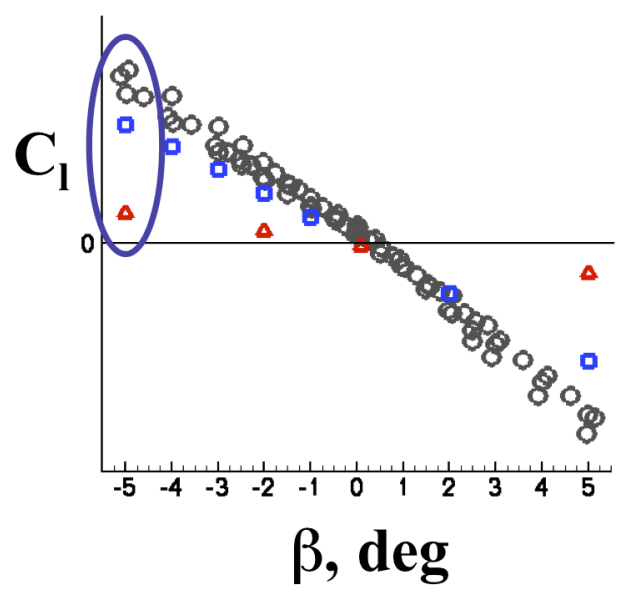

Wind Tunnel Data

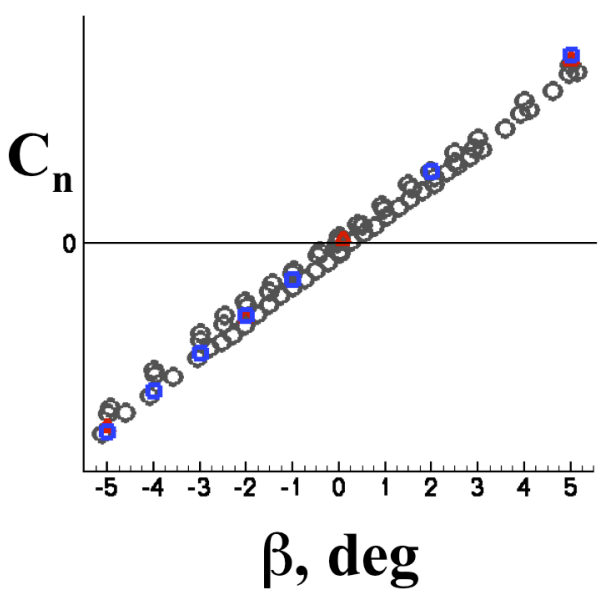

$\Delta$ USM3Dns (blind)

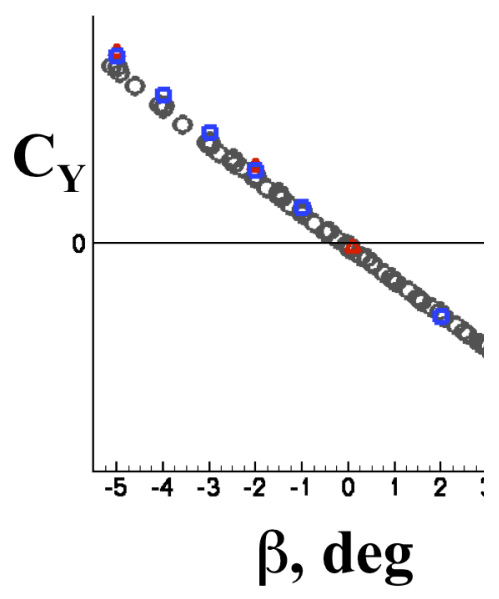

- USM3Dns $\left(2^{\text {nd }}\right.$ try)

Figure 24. F/A-18E in sideslip, $M_{\infty}=0.9, \operatorname{Re}_{c}=4.0$ million, $\alpha=10^{\circ}$. 


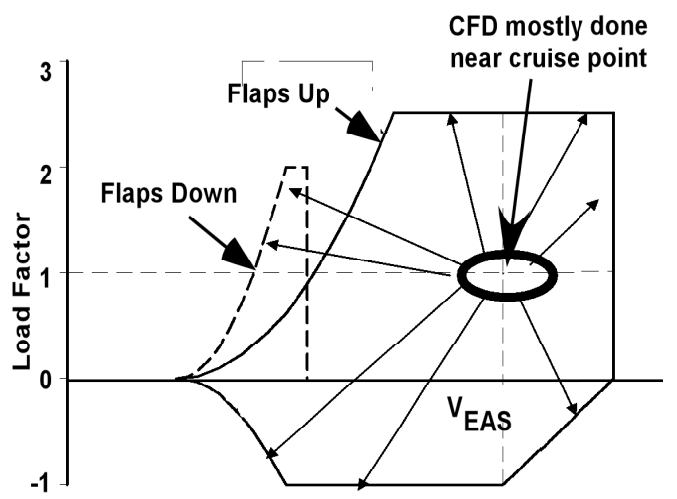

Figure 25. CFD and the flight envelope.

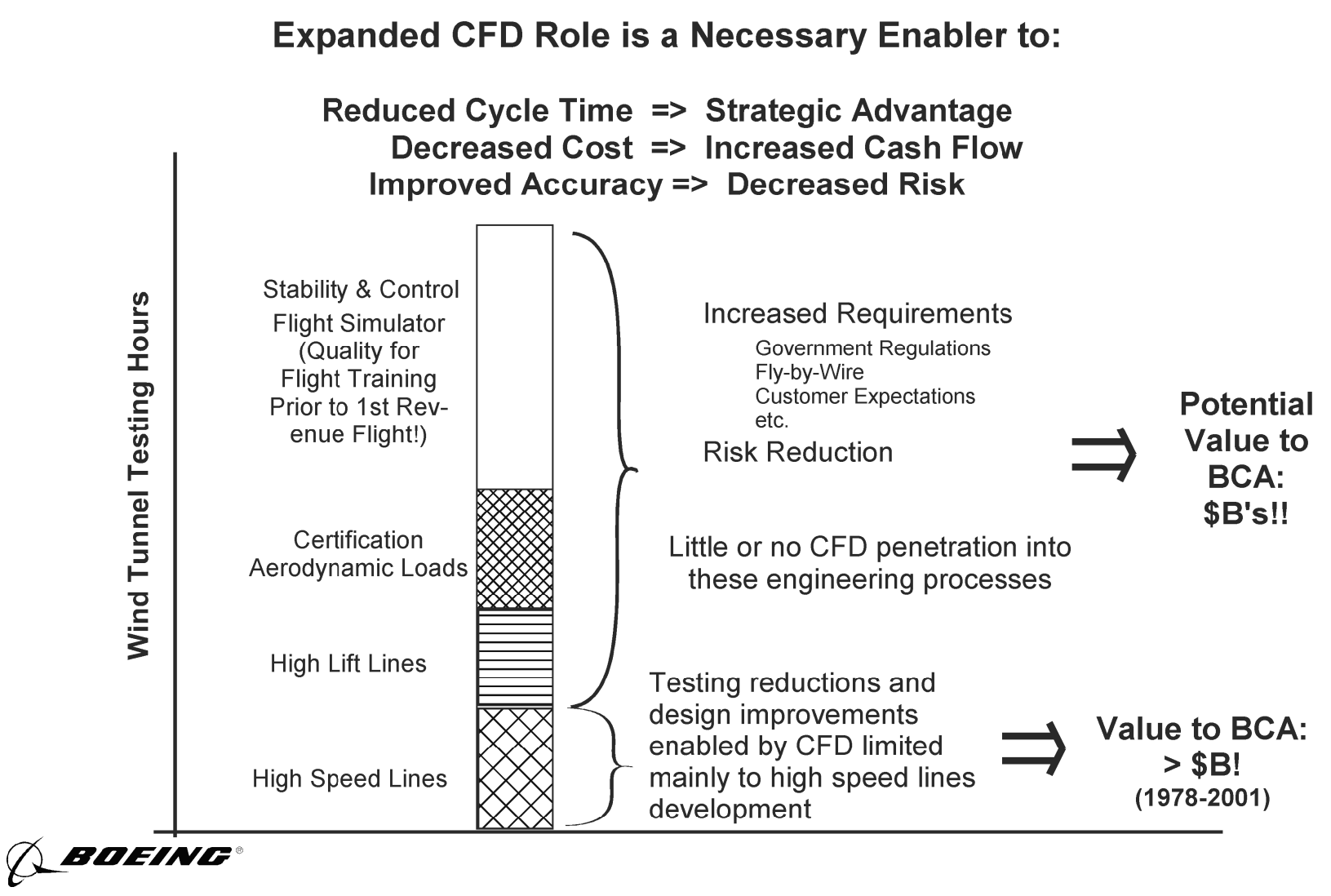

Figure 26. The opportunity to expand CFD usage and reduce wind tunnel testing hours. 


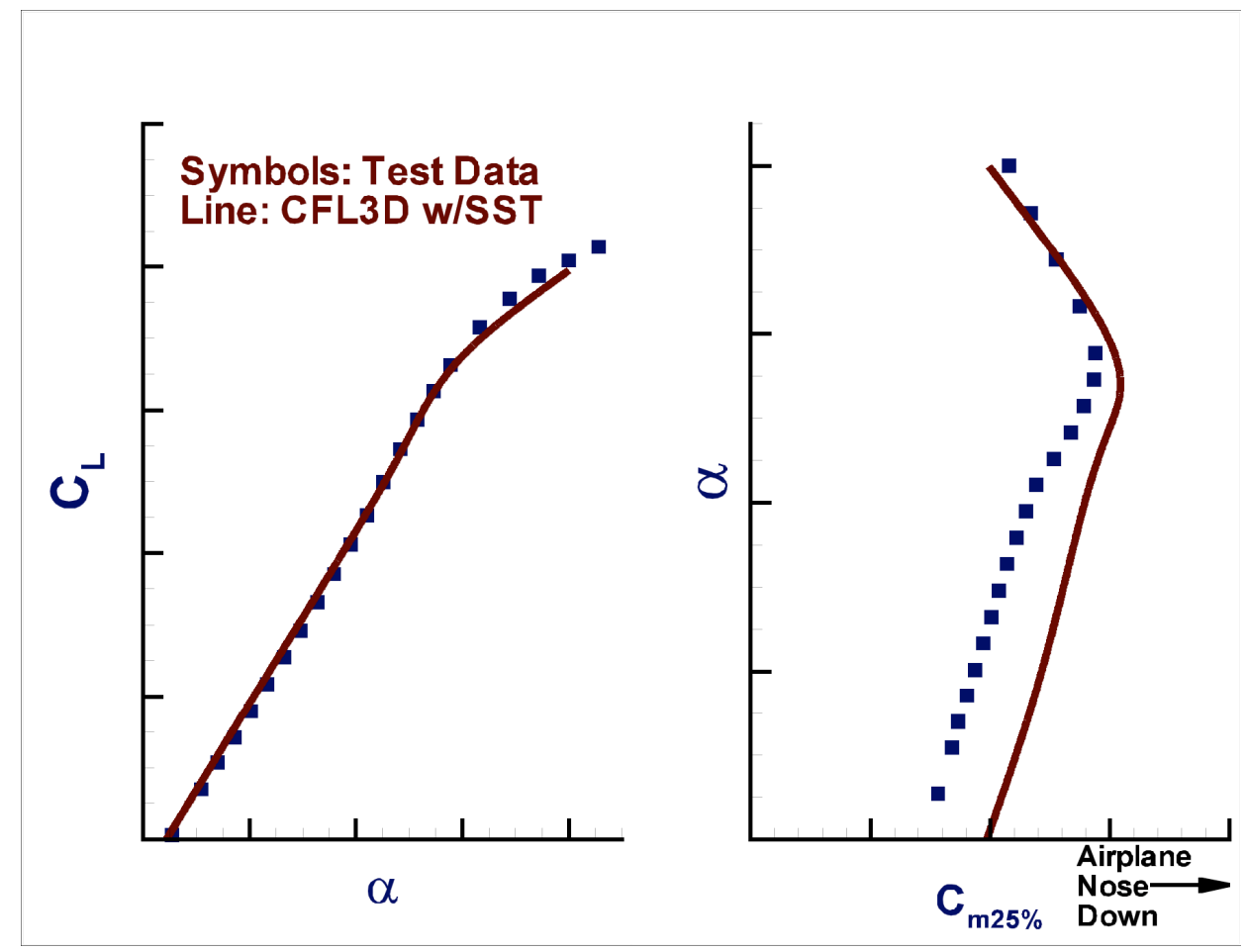

Figure 27. Pitch characteristics of a tail-off transonic transport.

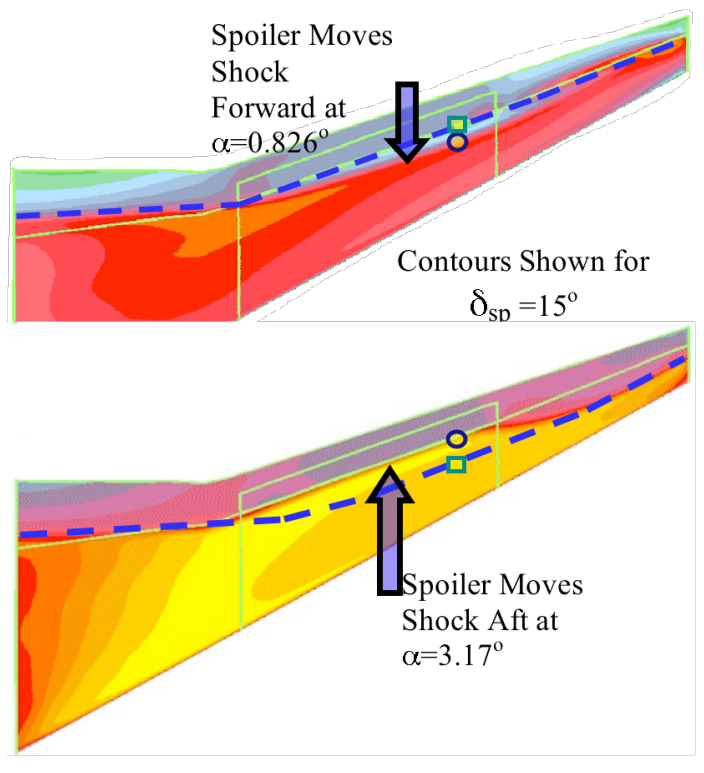

\section{Legend}

- - $-\delta_{\mathrm{sp}}=0^{\circ}$ CFD Shock Position Spoiler Planform

$\delta_{\mathrm{sp}}=15^{\circ} \mathrm{W}$.Tun. Shock Position

$\square \delta_{\mathrm{sp}}=0^{\circ}$ W.Tun. Shock Position

Figure 28. Transonic spoiler reversal. 


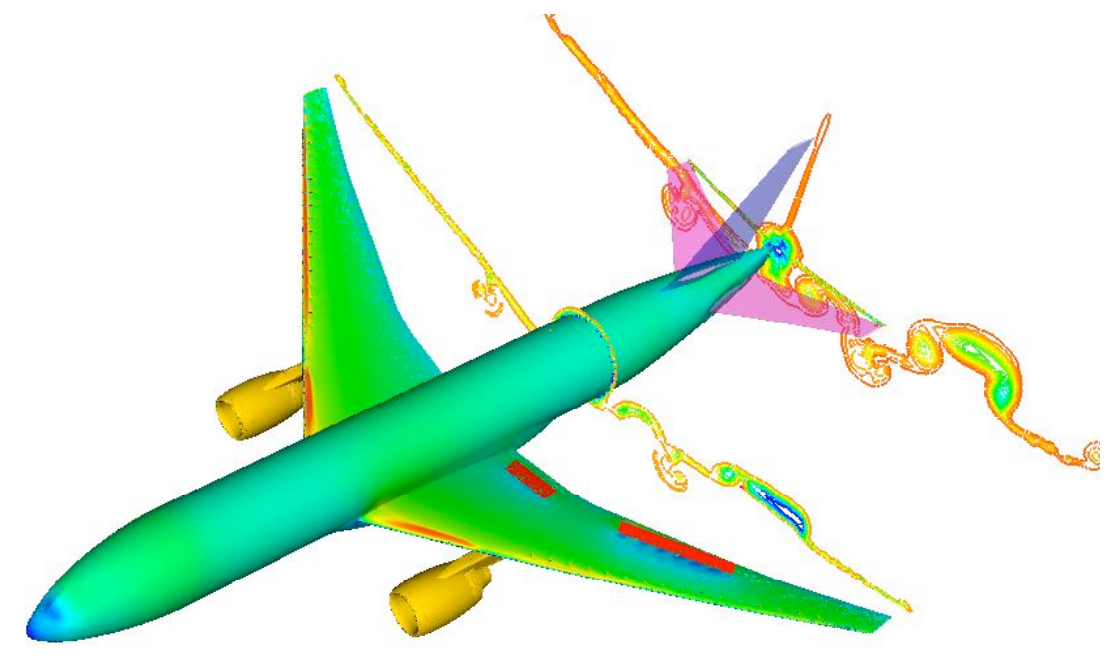

Figure 29. Transonic Navier-Stokes simulation with spoilers, which are in red.

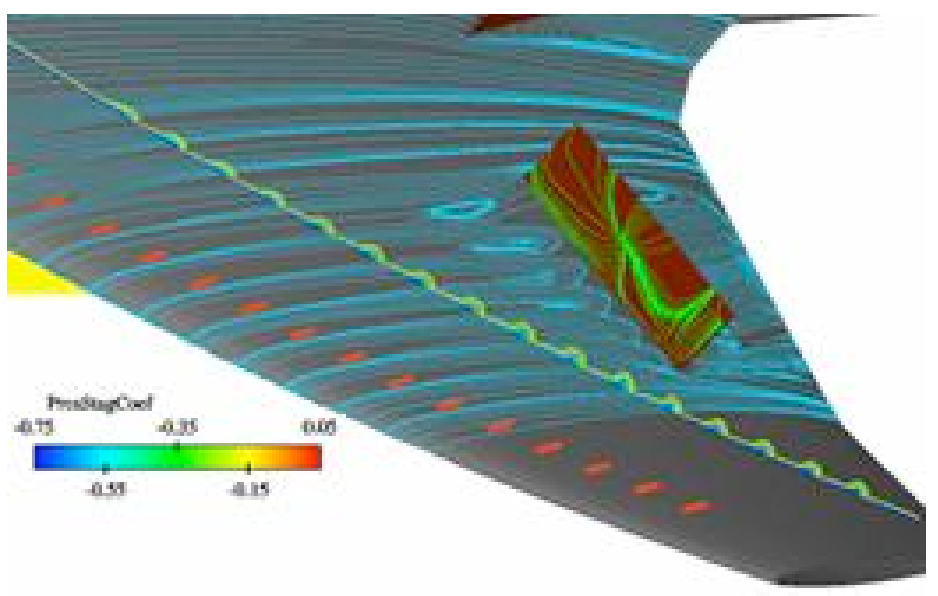

Figure 30. Flow detail near spoiler of figure 28.

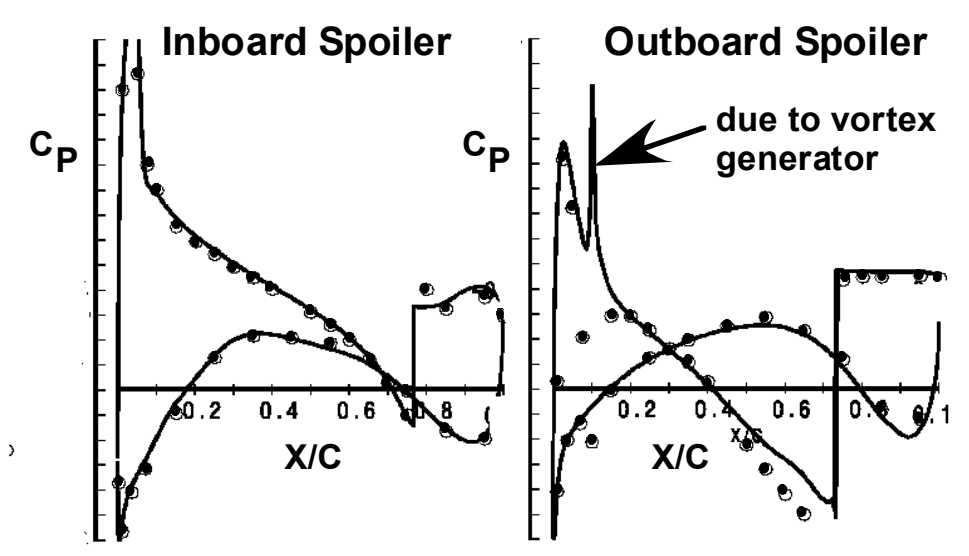

Figure 31. Pressure distribution with deflected spoiler. 


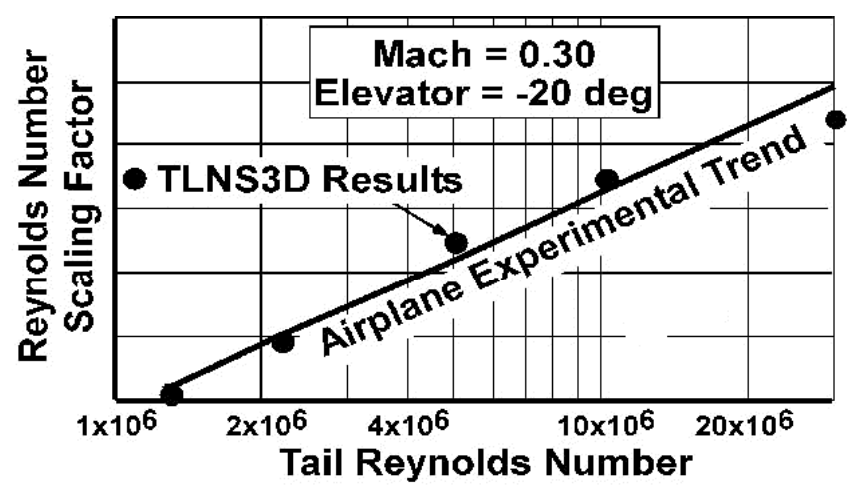

Figure 32. Elevator control effectiveness.

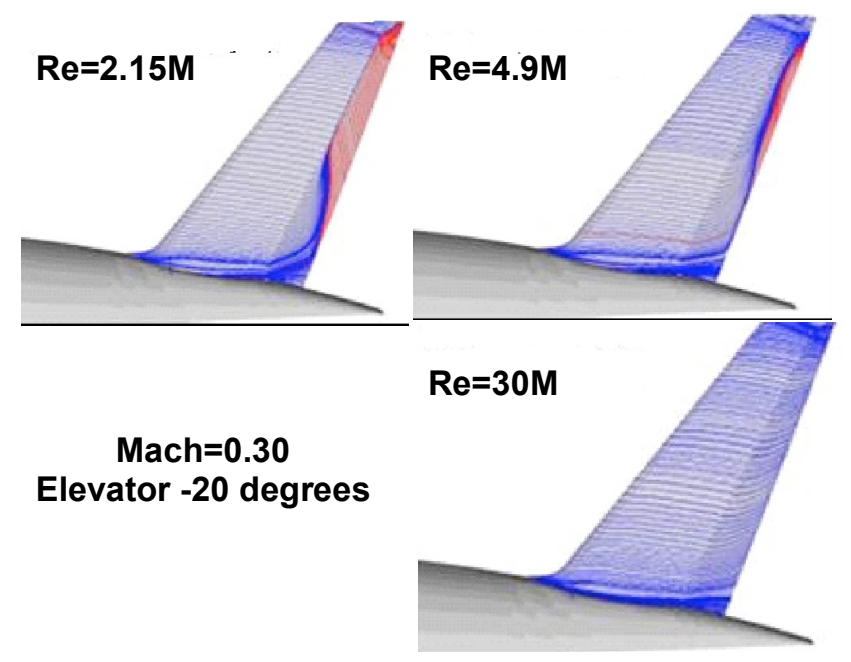

Figure 33. Reynolds number effects with deflected elevator.

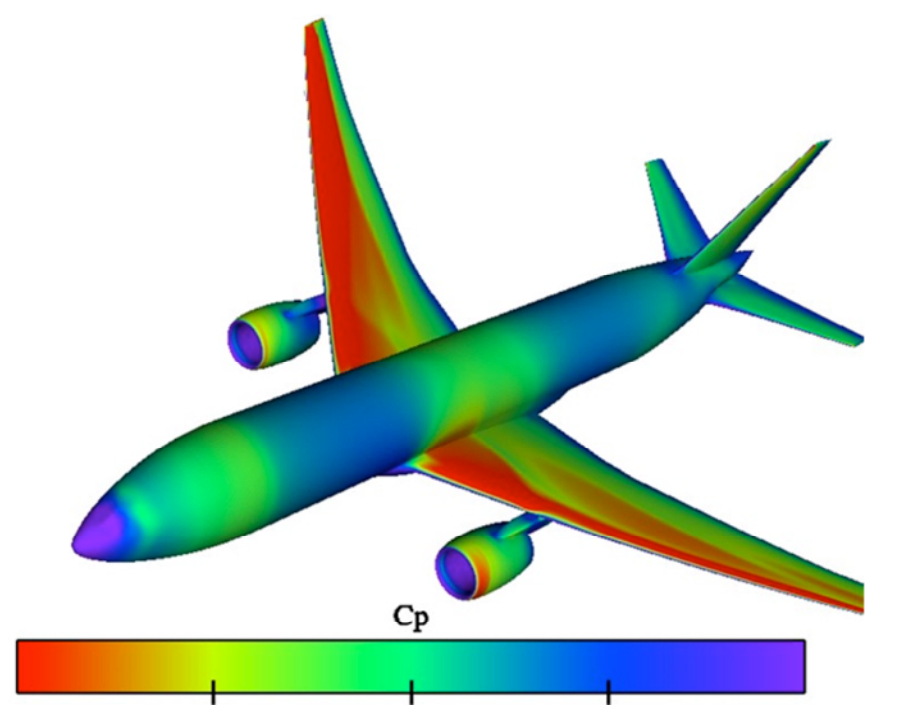

Figure 34. Transonic twinjet in sideslip. 


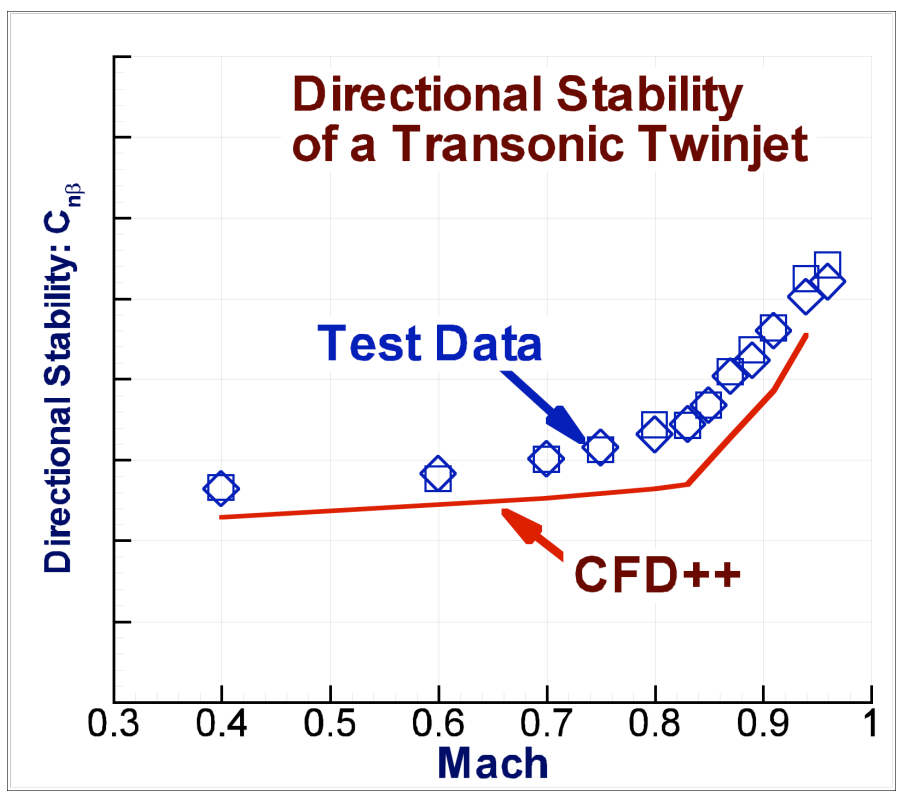

Figure 35. Directional stability for a transonic twinjet.

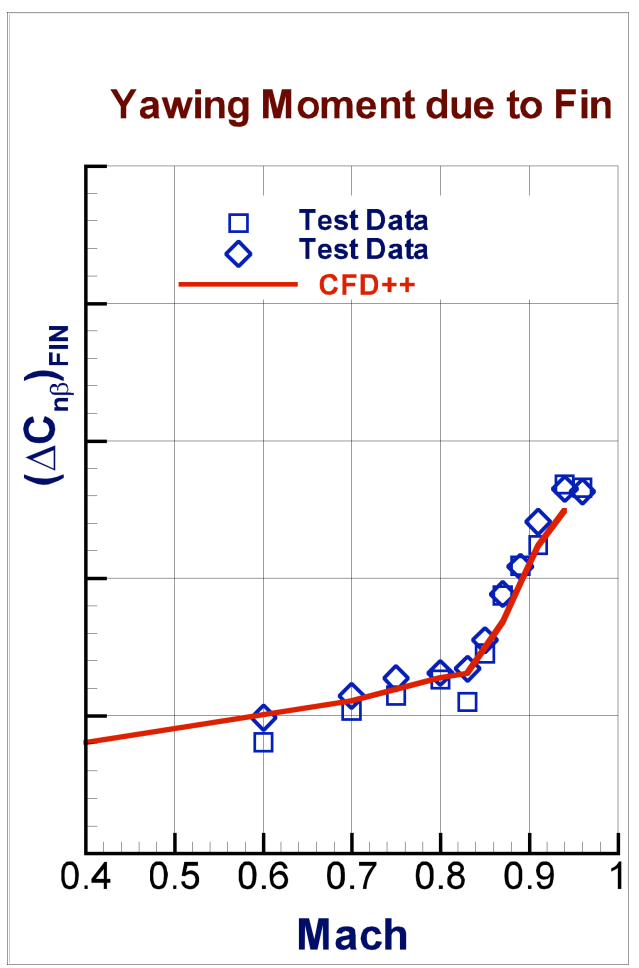

Figure 36. Fin contribution to directional stability. 

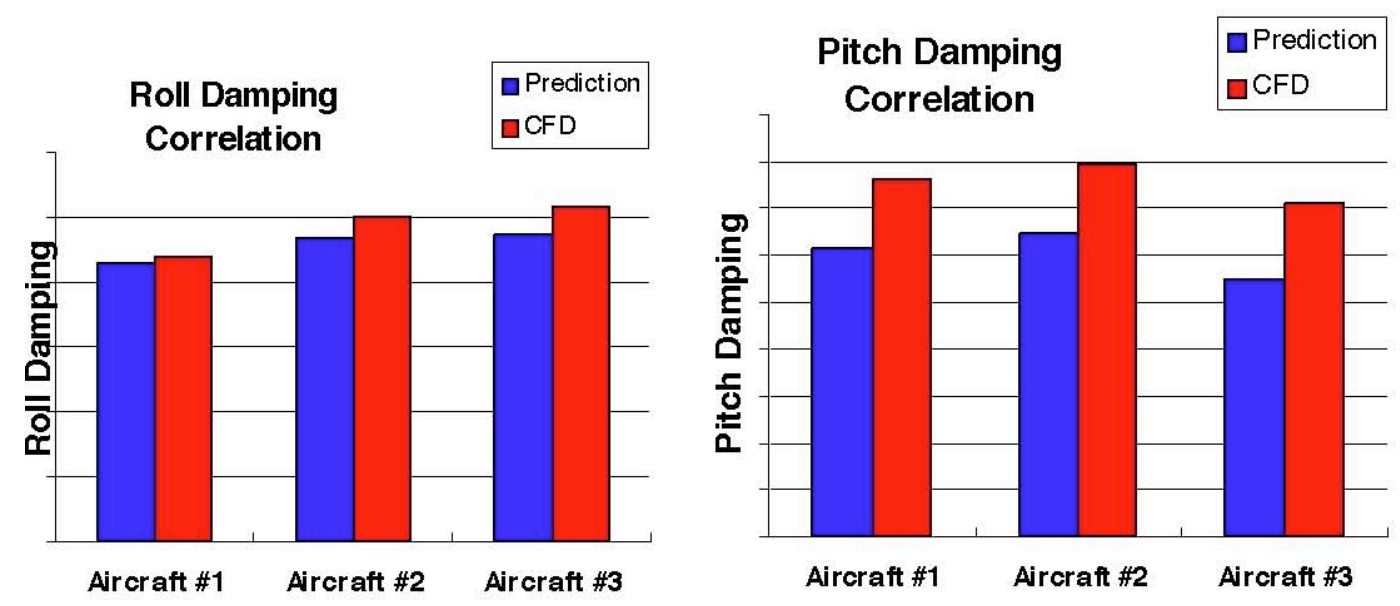

Figure 37. Dynamic derivative correlation between Panel Method (CFD) and handbook.

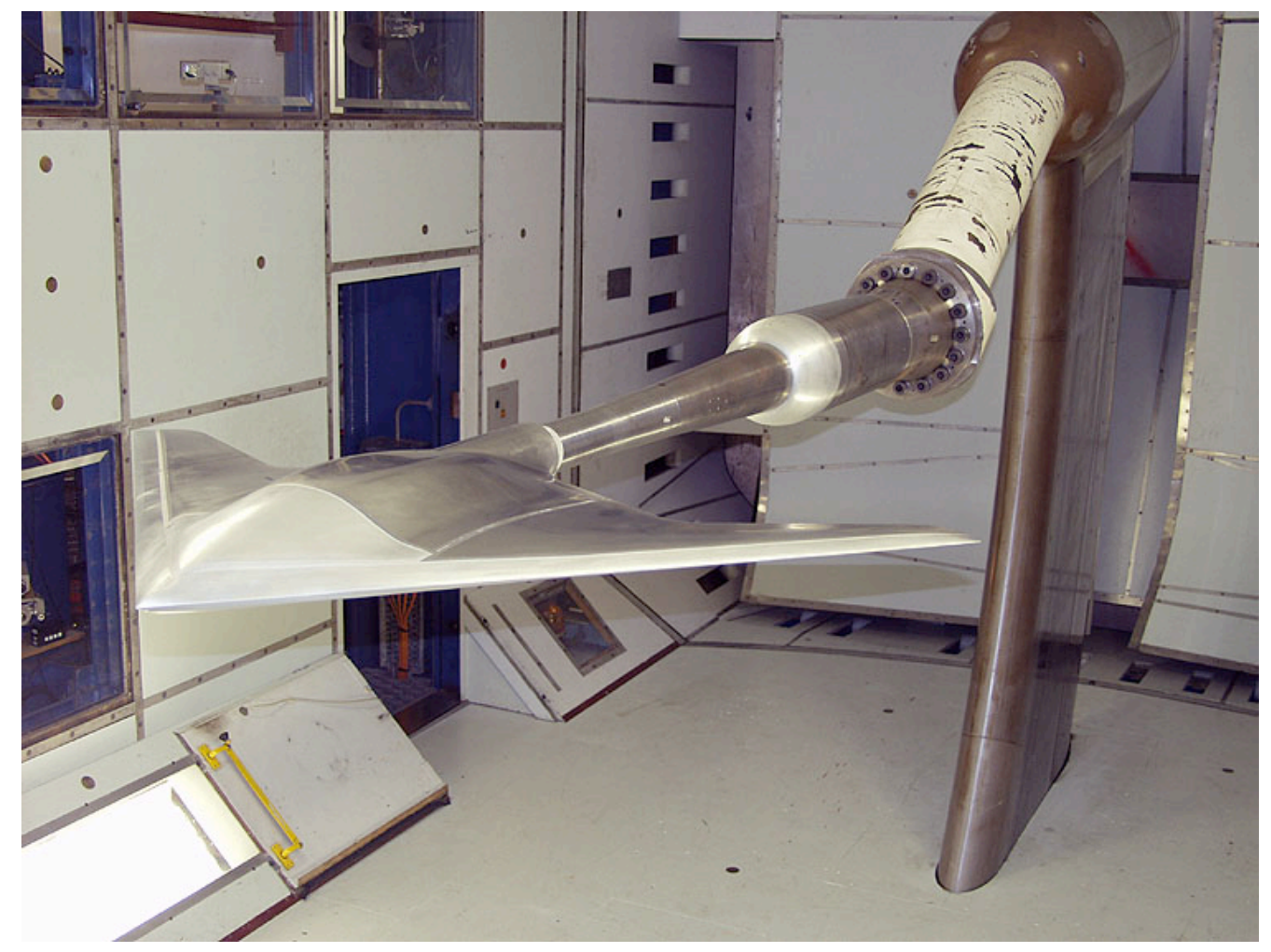

Figure 38. 1303 UCAV in 5m pressure tunnel at Farnborough, England, July 2002. 


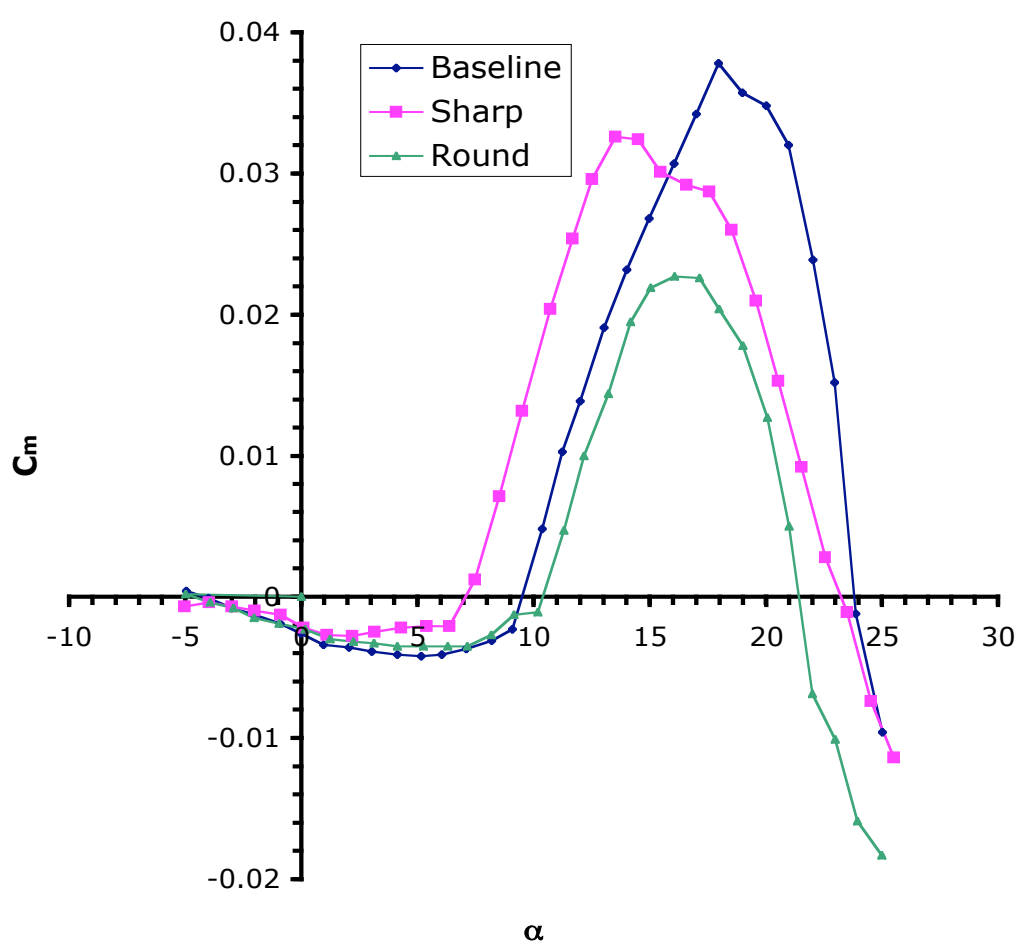

Figure 39. Nonlinear pitching moment for $1303 \mathrm{UCAV}$ with various leading edge geometries.

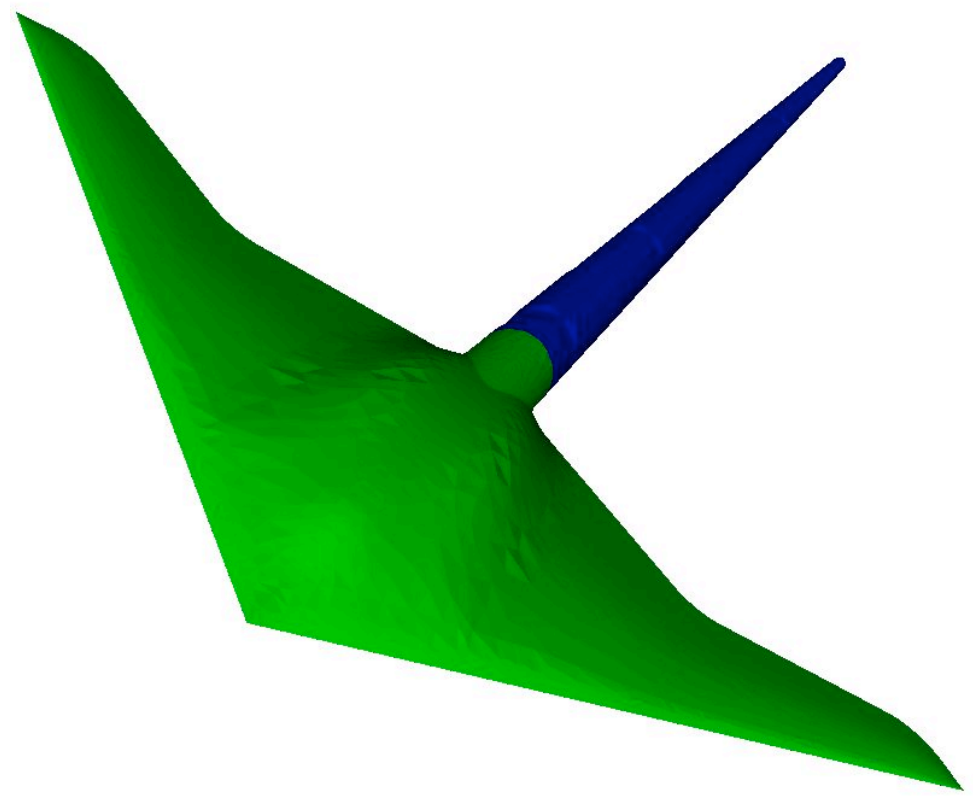

Figure 40. Initial 1303 geometry for CFD showing representative sting connected to model. 

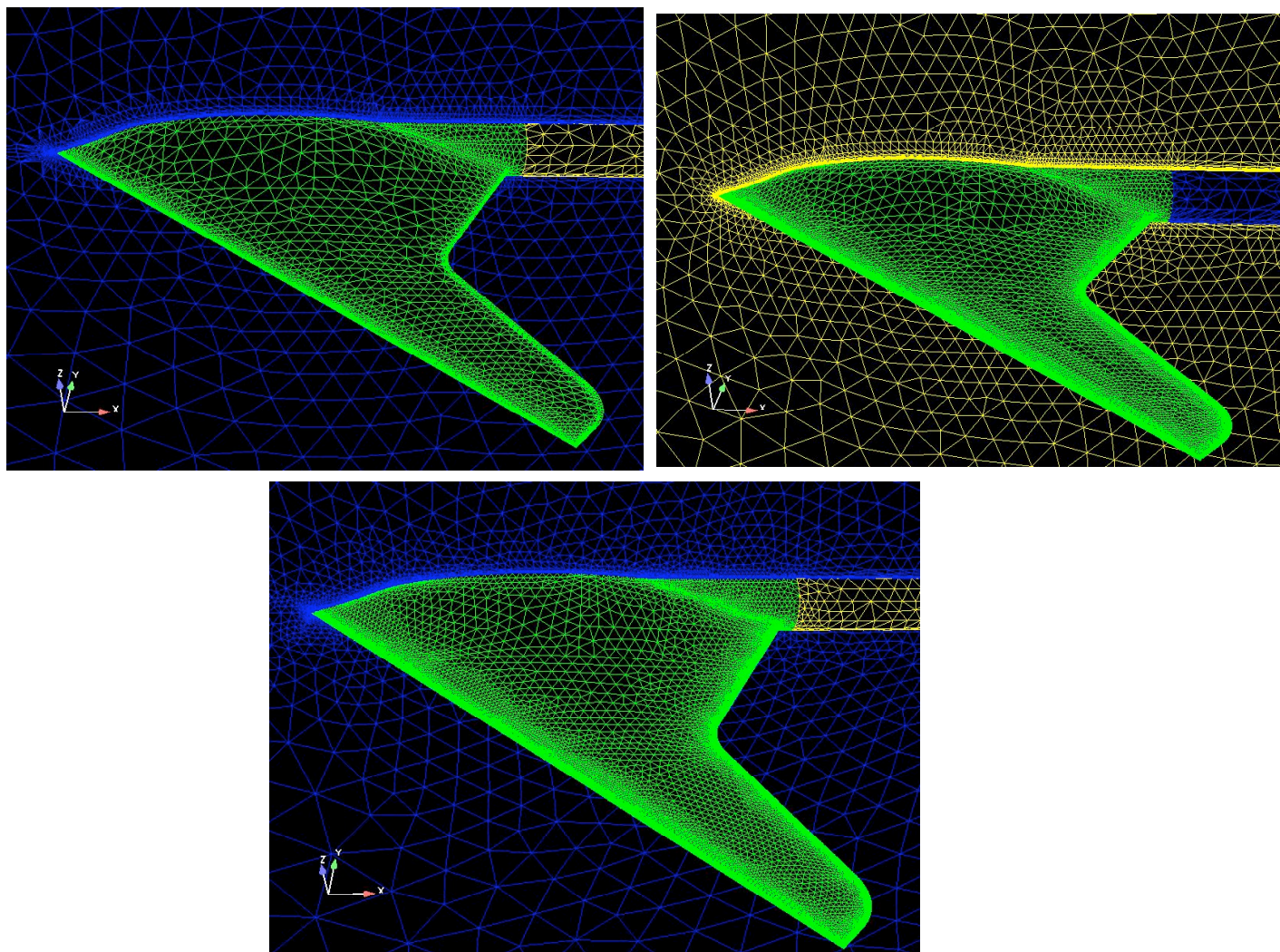

Figure 41. Surface triangulations for 1.7, 4.7, and 8.3 million cell grids (respectively) used in mesh refinement study.

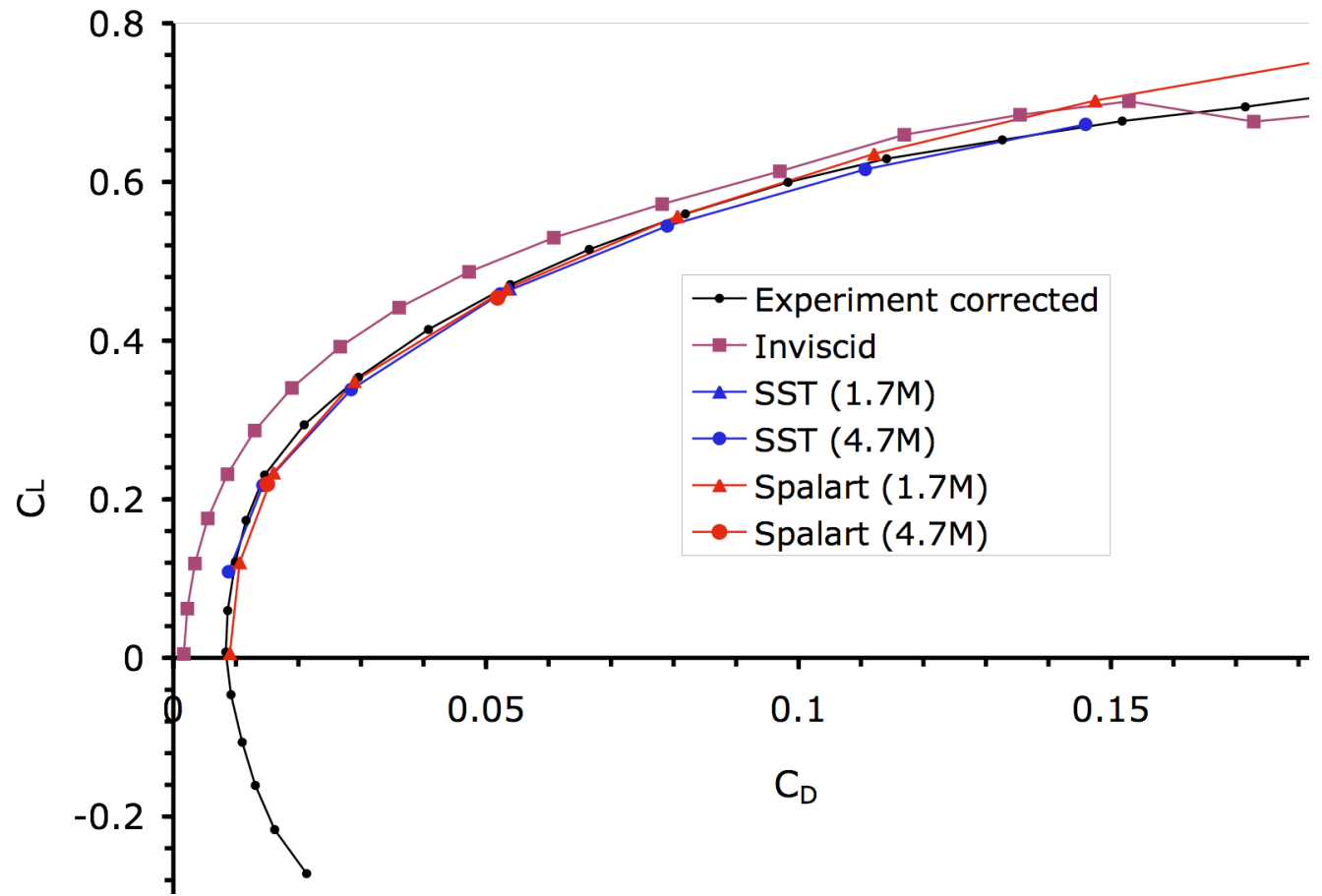

Figure 42. AVUS drag polar comparison with corrected experimental data for various grid resolutions and turbulence models $\left(\mathrm{M}_{\infty}=0.25, \mathrm{Re}=4.28\right.$ million $)$. 


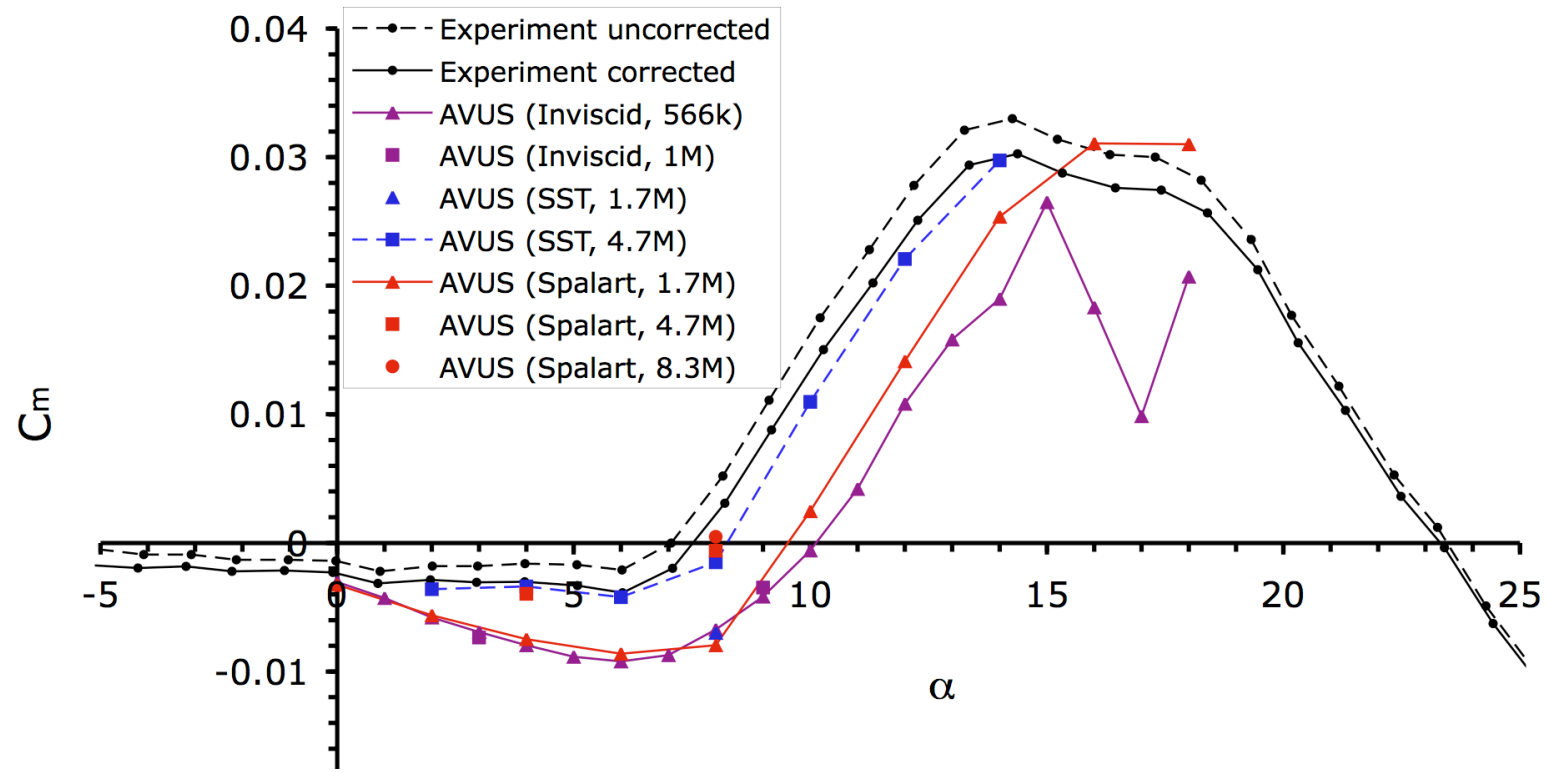

Figure 43. AVUS pitching moment comparison with corrected and uncorrected experimental data for various grids resolutions and turbulence models $\left(\mathrm{M}_{\infty}=0.25, \mathrm{Re}=4.28\right.$ million $)$.
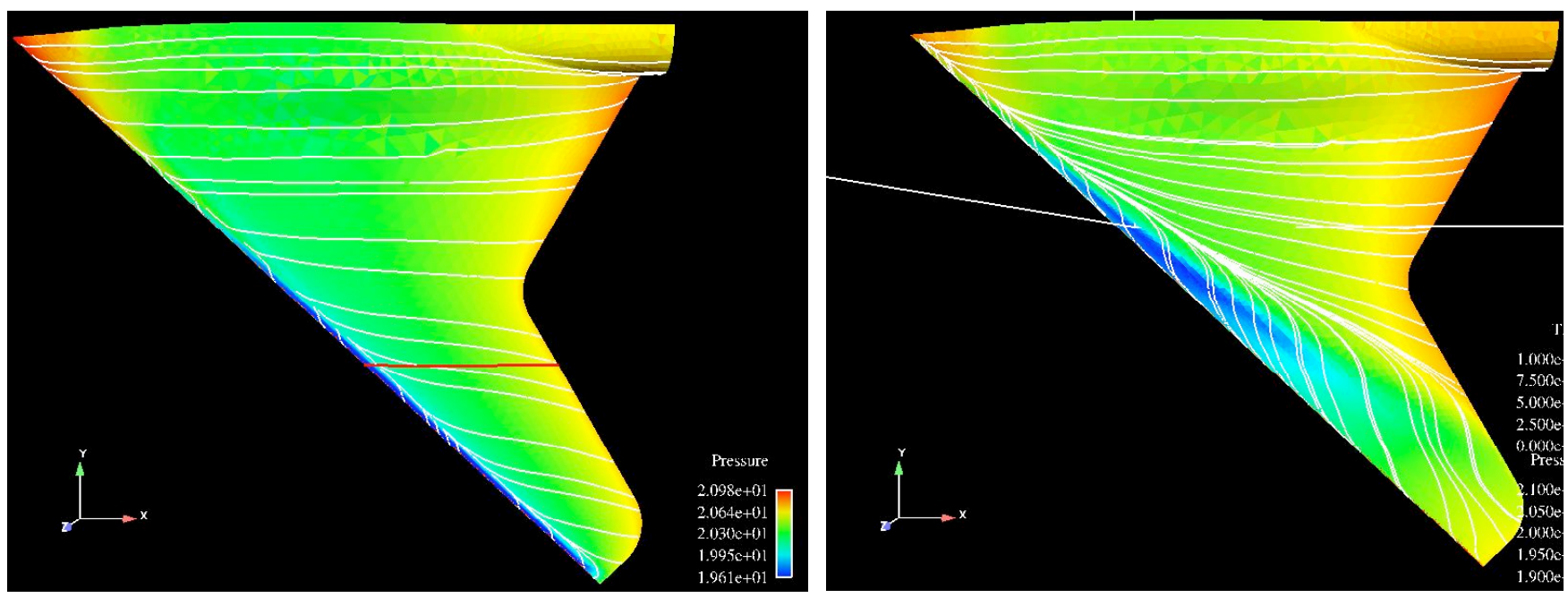

Figure 44. Surface oil flow and pressure contours on 4.7 million cell grid for solutions with Menter's SST turbulence model at $4^{\circ}$ and $8^{\circ}$ angle of attack, respectively $\left(M_{\infty}=0.25, \operatorname{Re}=4.28\right.$ million). 


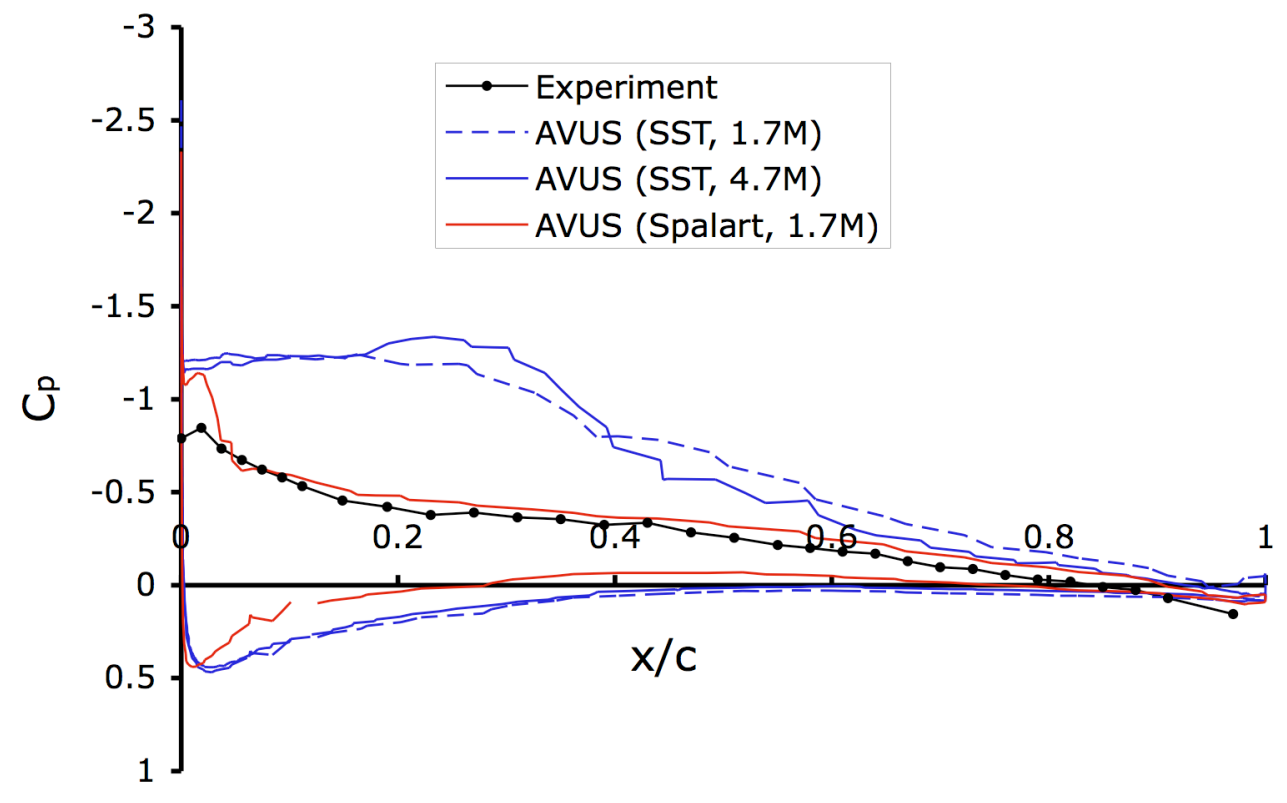

Figure 45. Computed pressure coefficient comparison with experiment at 18 -inch span location for $4^{\circ}$ angle of attack $\left(\mathrm{M}_{\infty}=0.25, \operatorname{Re}=4.28\right.$ million $)$.

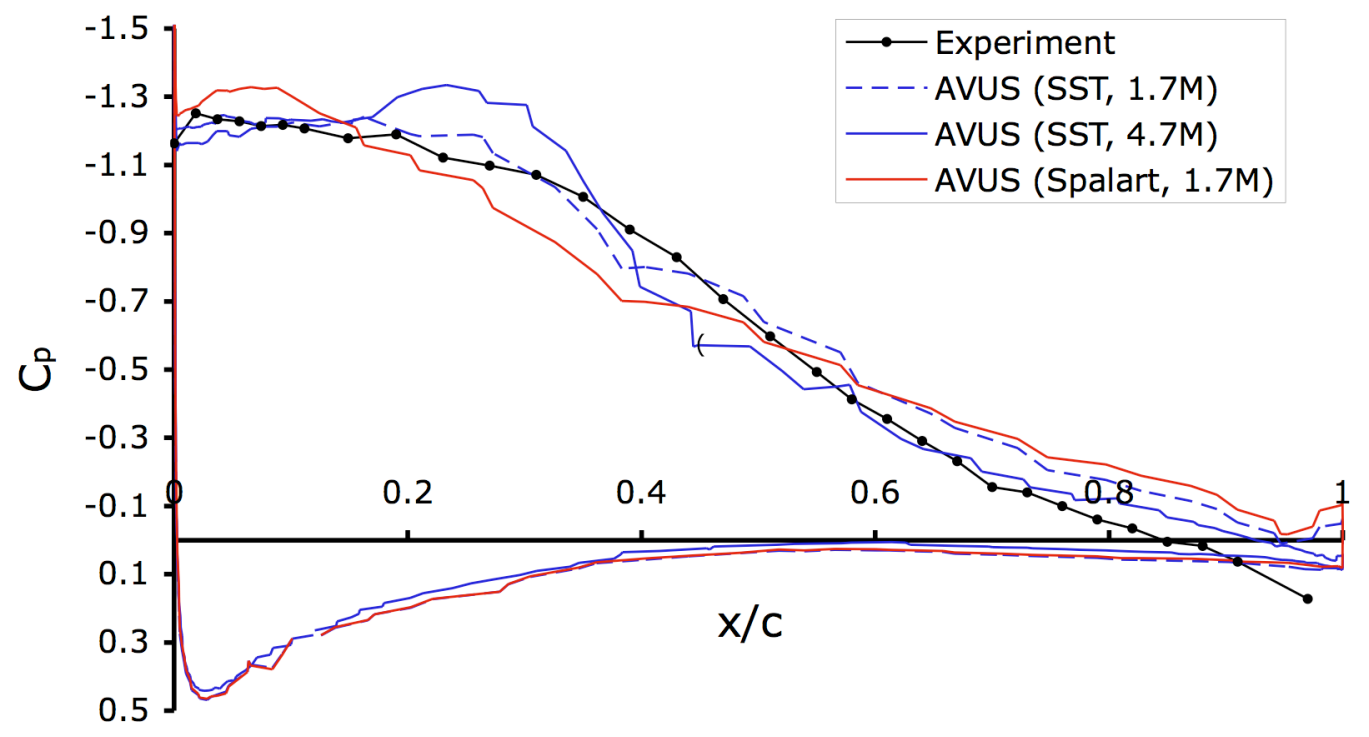

Figure 46. Computed pressure coefficient comparison with experiment at 18 -inch span location for $8^{\circ}$ angle of attack $\left(\mathrm{M}_{\infty}=0.25, \mathrm{Re}=4.28\right.$ million $)$. 